\title{
OLEDs for General Lighting
}

\author{
Final Report \\ Contract No. DE-FC26-00NT40989
}

\author{
Principal Investigator \\ Anil Duggal \\ GE Global Research
}

Project Team Members

Anil Duggal, Don Foust, Chris Heller, Bill Nealon, Larry Turner, Joe Shiang GE Global Research

Nick Baynes, Tim Butler, Nalin Patel

Cambridge Display Technology 


\section{Executive Summary}

The goal of this program was to reduce the long term technical risks that were keeping the lighting industry from embracing and developing organic light-emitting diode (OLED) technology for general illumination. The specific goal was to develop OLEDs for lighting to the point where it was possible to demonstrate a large area white light panel with brightness and light quality comparable to a fluorescent source and with an efficacy comparable to that of an incandescent source. It was recognized that achieving this would require significant advances in three areas - (a) the improvement of white light quality for illumination, (b) the improvement of OLED energy efficiency at high brightness, and (c) the development of cost-effective large area fabrication techniques.

The program was organized such that, each year, a "deliverable" device would be fabricated which demonstrated progress in one or more of the three critical research areas. In the first year (2001), effort concentrated on developing an OLED capable of generating high illumination-quality white light. Ultimately, a down-conversion method where a blue OLED was coupled with various down-conversion layers was chosen. Various color and scattering models were developed to aid in material development and device optimization. The first year utilized this approach to deliver a 1"x1" OLED with higher illumination-quality than available fluorescent sources. A picture of this device is shown in Figure E1 and performance metrics are listed in Table E1. To our knowledge, this was the first demonstration of true illumination-quality light from an OLED.

During the second year, effort concentrated on developing a scalable approach to large area devices. A novel device architecture consisting of dividing the device area into smaller elements that are monolithically connected in series was developed. In the course of this development, it was realized that, in addition to being scalable, this approach made the device tolerant to the most common OLED defect - electrical shorts. This architecture enabled the fabrication of a 6"x6" OLED deliverable for 2002. A picture of this deliverable is shown in Figure E2 and, again, the performance metrics are listed in Table E1. At the time, this was the highest efficiency, highest lumen output illumination-quality OLED in existence.

The third year effort concentrated on improving the fabrication yield of the 6"x6" devices and improving the underlying blue device efficiency. An efficiency breakthrough was achieved through the invention of a new device structure such that now 15 lumen per watt devices could be fabricated. A 2'x2' OLED panel consisting of sixteen 6" 6 " high efficiency devices tiled together was then fabricated. Pictures of this panel are shown in Figure E3 with performance metrics in Table E1. This panel met all project objectives and was the final deliverable for the project. It is now the highest efficiency, highest lumen output, illumination-quality OLED in existence. 
Table E1: OLED “deliverable" device performance.

\begin{tabular}{lccc}
\multicolumn{1}{c}{ Attribute } & $\mathbf{2 0 0 1}$ & $\mathbf{2 0 0 2}$ & $\mathbf{2 0 0 3}$ \\
\hline \hline Size $\left(\right.$ in $\left.^{2}\right)$ & 1 & 36 & 576 \\
Output (Lumens) & 2 & 70 & 1200 \\
Efficacy (Lumens/watt) & 4 & 7 & 15 \\
Color Temperature (K) & 93 & 90 & 88 \\
Color Rendering Index & 4100 & 4000 & 4000
\end{tabular}


Figure E1: 2001 - 1"x1" OLED deliverable.

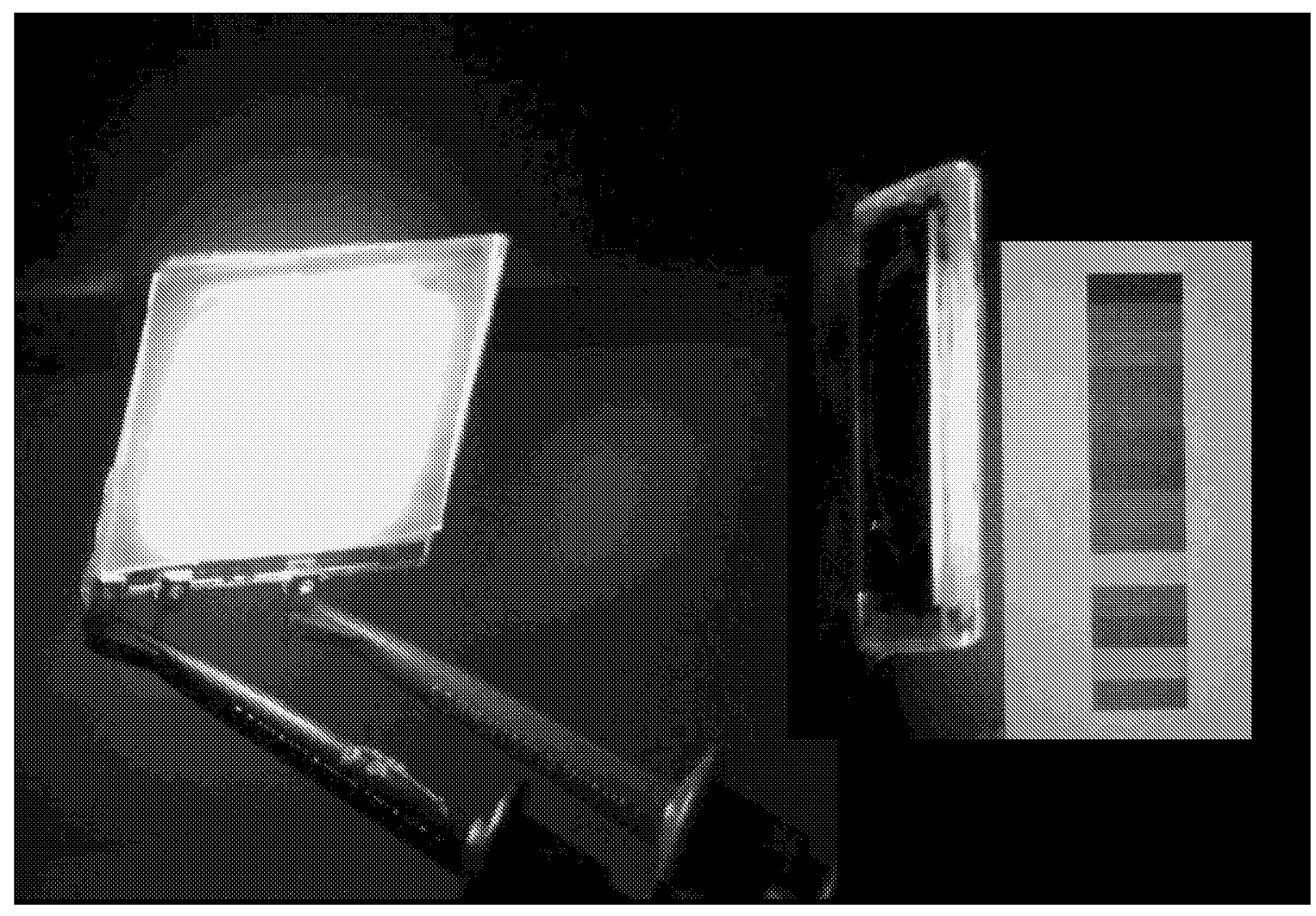


Figure E2: 2002 - 6"x6" OLED deliverable.

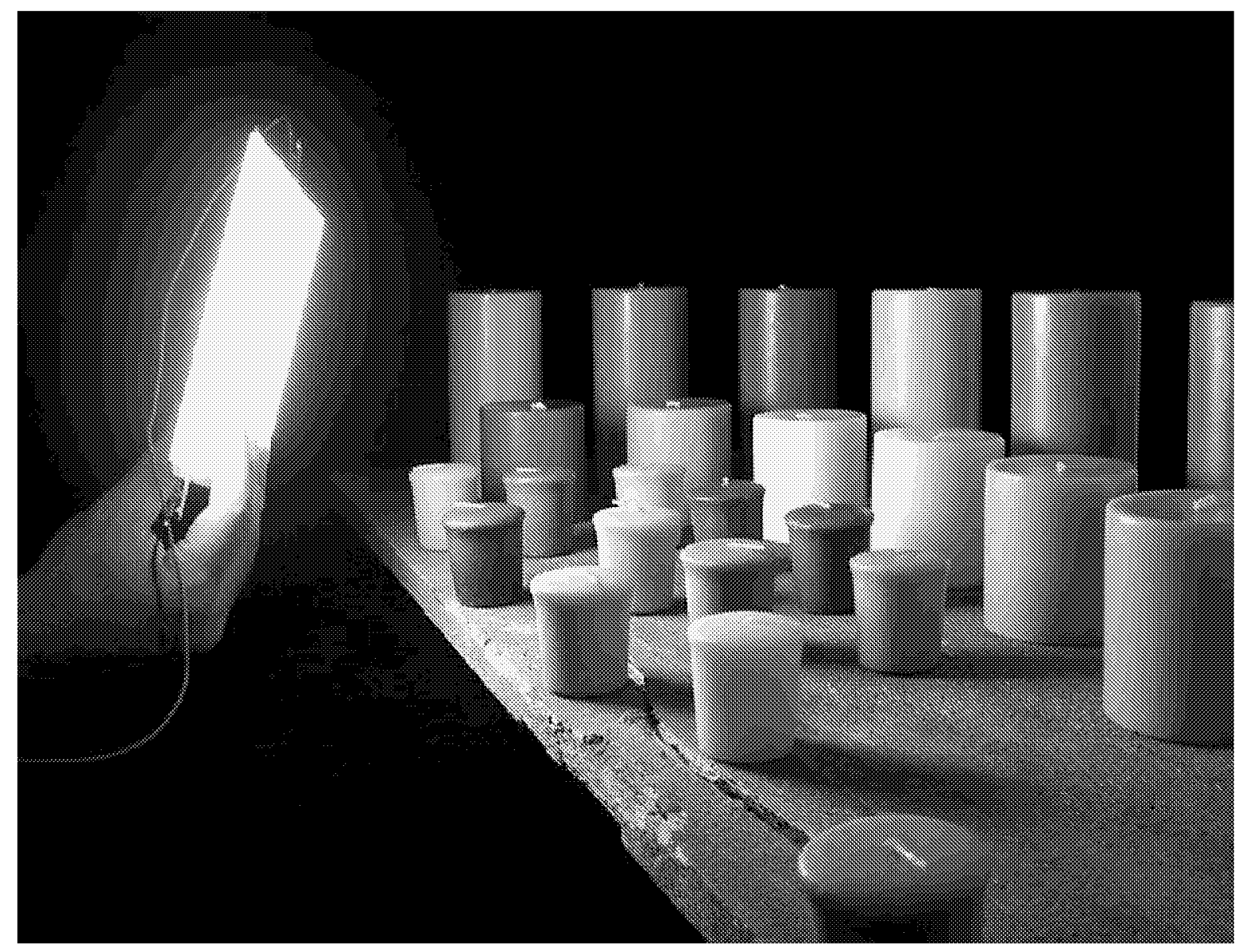


Figure E3: 2003 -2'x2' OLED lighting panel deliverable.
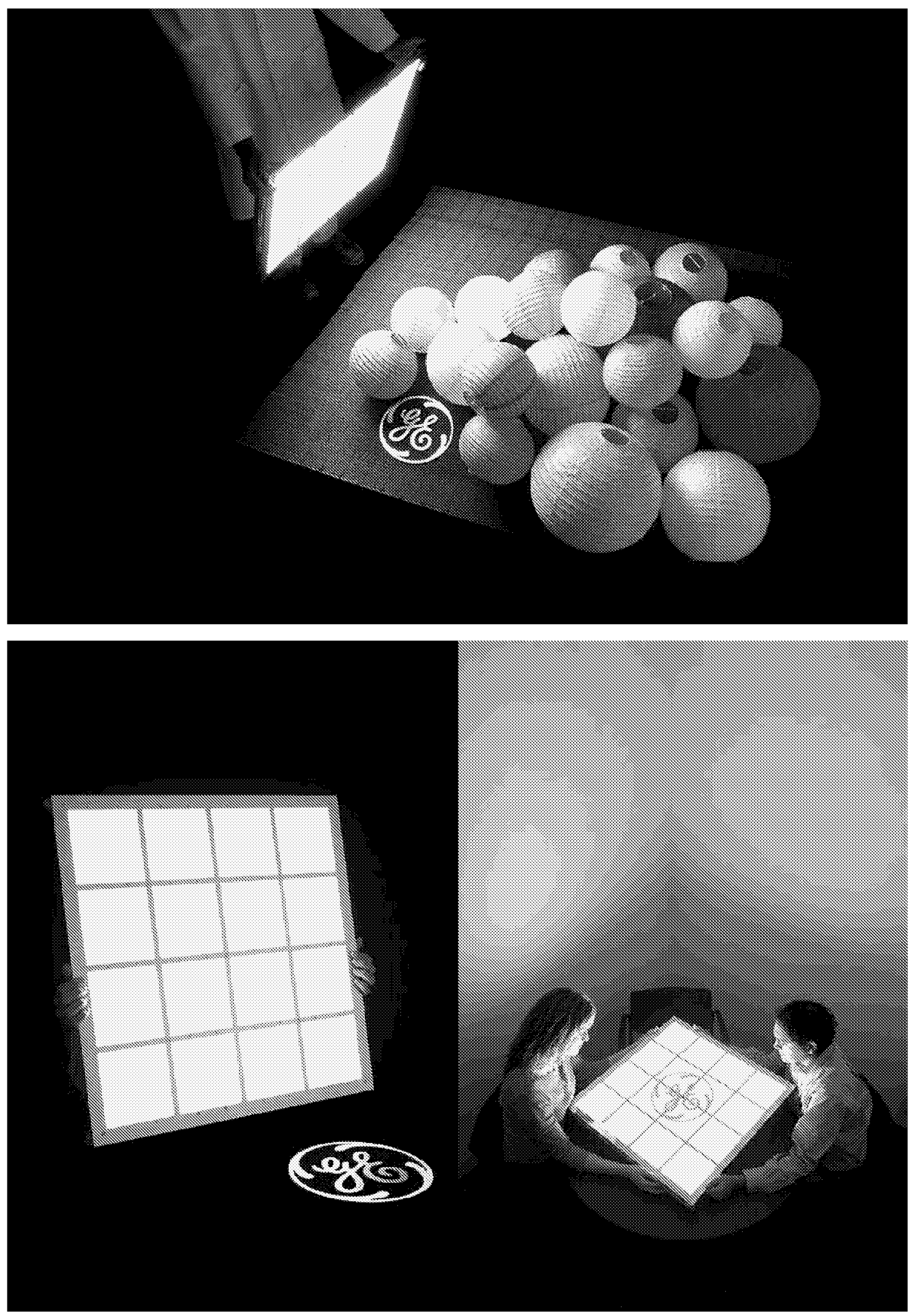


\section{Introduction}

The goal of this program was to reduce the long term technical risks that were keeping the lighting industry from embracing and developing organic light-emitting diode (OLED) technology for general illumination. The specific goal was to develop OLEDs for lighting to the point where it was possible to demonstrate a large area white light panel with brightness and light quality comparable to a fluorescent source and with an efficacy comparable to that of an incandescent source. As described in the Executive Summary, this specific goal was successfully achieved at the end of the program.

This report describes the technical details of the advances that were made in three key areas. Section II describes the method for creating illumination quality white light. Section III gives a detailed theoretical and experimental analysis of the use of light scattering to increase the efficiency of OLED devices and Section IV describes the novel architecture that was developed to enable scalable, fault-tolerant OLEDs.

\section{Creating Illumination-Quality White Light ${ }^{1}$}

From the lighting perspective, light quality refers primarily to the color and the color rendition index (CRI) of the light. For illumination applications, the color needs to be equivalent to that of a blackbody source between 3000 and $6000 \mathrm{~K}$. The allowable colors expressed in terms of the commonly used CIE $\mathrm{x}$ and $\mathrm{y}$ coordinates can be found in standard texts ${ }^{2}$. The colors of typical light sources fall within $0.01 \mathrm{x}$ or $\mathrm{y}$ units of the exact blackbody source color. The CRI is a numerical measure of how "true" colors look when viewed with the light source. It can be determined quantitatively from the output spectrum of the light source ${ }^{2}$. The CRI is defined such that a blackbody source has the highest value of 100 and all other sources have lower values. High illumination quality sources typically have CRI values greater than 80 . For commercial viability, the color and CRI of a light source need to be stable over source lifetime.

Numerous methods of making an intrinsically white emitting OLED by blending different emissive species into the emissive region - either in a single layer or in multiple layers have been demonstrated ${ }^{3,4,5}$. With proper care of spectra and concentrations of the component emissive species these methods could potentially provide illumination quality white light. However, all these methods are likely to have difficulty with color stability due to differential aging of the various emitting species. In this letter we demonstrate an alternative method for making a white OLED which should be far more robust to color shifting as electroluminescence intensity decreases with age. The method consists of coupling a blue-emitting OLED utilizing a single emissive species with one or more down-conversion layers - one of which contains inorganic scattering particles. We demonstrate that this method can provide illumination quality light with no efficiency loss from the down-conversion process. 
The white device design consists of a blue light emitting polymer (LEP) - based OLED with a $6.35 \mathrm{~cm}^{2}$ active area deposited on a glass substrate, and on the reverse side of the glass, a series of down-conversion layers which consist of organic molecules (perylene orange, perylene red) and inorganic phosphor particles (Y(Gd)AG:Ce). The device was fabricated as follows. Indium tin oxide (ITO) coated glass obtained from Applied Films Corporation was patterned using standard photolithography techniques and cleaned with an oxygen plasma. A thin $(60 \mathrm{~nm})$ film of PEDOT/PSS (Poly $(3,4)-$ ethylendioxythiophene / polystyrene sulfonate) - polymer obtained from Bayer Corporation was immediately deposited atop the ITO via spin casting and then baked for one hour at $200{ }^{\circ} \mathrm{C}$. A polyfluorene-based blue LEP obtained from Cambridge Display Technologies (Cambridge, England) was then spin coated atop the PEDOT/PSS layer to a thickness of about $60-70 \mathrm{~nm}$. A cathode consisting of $4 \mathrm{~nm}$ of NaF followed by $200 \mathrm{~nm}$ of $\mathrm{Al}$ was then thermally evaporated atop the blue LEP and then a top encapsulating cover glass was sealed to the device in an inert atmosphere. The organic dyes, Lumogen ${ }^{\mathrm{TM}} \mathrm{F}$ orange and red, obtained from BASF AG of Germany, were dispersed into thins films of polymethylmethacrylate ("PMMA", index of refraction, $\mathrm{n}=1.49$ ). Y(Gd)AG:Ce phosphor particles prepared at General Electric was dispersed in a poly-dimethyl siloxane (PDMS) silicone $(\mathrm{n}=1.41)$. The phosphor layers were optically coupled to the glass and each other using 25 micron layers of optical laminating tape $(3 \mathrm{M} \# 8141, \mathrm{n}=1.49)$ in the following order: OLED, orange, red, Y(Gd)AG:Ce. The quantum efficiency of the organic dyes in solution is $>0.98$ and no loss in efficiency was observed with a PMMA host. The quantum yield of the $\mathrm{Y}(\mathrm{Gd}) \mathrm{AG}$ :Ce was measured relative to a known standard and found to be 0.86 .

The measured EL spectra of the blue device and the absorption and emission spectra of each down conversion component are shown in Figure 1. The thickness and concentration of each down-conversion layer was chosen such that the final mixture of down-converted and unabsorbed light from each layer yields a white color. The output spectrum of the resulting white device is shown in the bottom panel of Figure 1. A color temperature of $4130 \mathrm{~K}$ on the blackbody locus and a CRI of 93 are calculated from this spectrum. These values indicate high illumination quality white light.

The brightness vs. voltage characteristics of the white and the underlying blue device are shown in Figure 2. The data was acquired by first measuring a blue device and then immediately applying the three phosphor layers and measuring the white light output. At $5.5 \mathrm{~V}$ the white device exhibits a brightness and luminous efficacy of 1080 $\mathrm{cd} / \mathrm{m}^{2}$ and 3.76 LPW while the underlying blue device exhibits $490 \mathrm{~cd} / \mathrm{m}^{2}$ and $1.73 \mathrm{LPW}$. We typically observe small run to run differences in the measured output of the blue devices, and the ratio of blue to white performance had a standard deviation of $6 \%$ between devices and measurements. In order to compare efficiency performance of the white and underlying blue device in a meaningful way it is necessary to convert from photometric units to radiometric units. This is accomplished by dividing the lumen values by the luminous efficacy (lumens per radiant watt) calculated from the spectra. The resultant efficiency curves as a function of voltage are shown in lower panel of Figure 2. Note that the power efficiency is larger for the white device. 
A simple model was developed to describe phosphor down-conversion. In the model, each phosphor layer absorbs a fraction of the input photons and emits them at a different wavelength. Thus the output of the device (in photons) upon exiting the $n^{\text {th }}$ phosphor layer is given by the following equation:

$$
\mathrm{S}_{\mathrm{n}}(\lambda)=\mathrm{S}_{\mathrm{n}-1}(\lambda) \exp \left(-\alpha_{\mathrm{n}}(\lambda) \delta_{\mathrm{n}}\right)+\mathrm{W}_{\mathrm{n}} \mathrm{C}_{\mathrm{n}}(\lambda) \mathrm{P}_{\mathrm{n}}(\lambda)
$$

where the first and second term describe the absorption and emission, respectively, in layer $n$. Here, $S_{0}(\lambda)$ is the output spectrum of the OLED, $\alpha_{n}(\lambda)$ is the absorption coefficient of the phosphor material in the $\mathrm{n}^{\text {th }}$ layer, and $\delta_{\mathrm{n}}$ is the effective optical path length. This length is different from the layer thickness due to scattering and non-normal propagation. The phosphor emission, $P_{n}(\lambda)$ is normalized so that its integral over all wavelengths is unity. This is multiplied by a weight factor, $\mathrm{W}_{\mathrm{n}}$, given by:

$$
W_{n}=Q_{n} \int S_{n-1}(\lambda)\left(1-\exp \left(-\alpha_{n}(\lambda) \delta_{n}\right)\right) d \lambda
$$

where $Q_{n}$ is the quantum yield and a self absorption correction $C_{n}(\lambda)$, given by ${ }^{6}$ :

$$
C_{n}(\lambda)=\frac{\exp \left(-\alpha_{n}(\lambda) \delta_{n}\right)}{1-Q_{n} \int P_{n}(\lambda)\left(1-\exp \left(-\alpha_{n}(\lambda) \delta_{n}\right)\right) d \lambda}
$$

Here, we have assumed that the effective path lengths for the self absorption process are equal to the effective path lengths for the luminescence process.

The model was used to fit the white output spectrum using the effective path lengths and an overall amplitude factor as adjustable parameters. Note that the values for $\mathrm{S}_{0}(\lambda), \mathrm{P}_{\mathrm{n}}(\lambda)$, and $\mathrm{Q}_{\mathrm{n}}$ were experimentally measured and fixed during fitting. The resulting fit, shown in Figure 1, is quite good given the simplicity of the model. The model appears robust as similar quality fits are obtained on devices where the concentration of the dye components was varied by up to a factor of 4 .

A prediction of the model is that by varying the effective absorption lengths of the different phosphor layers, any color from $3000-6000 \mathrm{~K}$ with a CRI of $>80$ can be generated using the same blue OLED. The model can also be used to estimate the ratio of white to blue power efficiency:

$$
\frac{P_{\text {white }}}{P_{\text {blue }}}=\frac{\int\left(S_{n}(\lambda) / \lambda\right) d \lambda}{\int\left(S_{0}(\lambda) / \lambda\right) d \lambda}
$$

According to the model, this ratio should always be less than one due to the finite quantum yields of the down-conversion layers and due to the fact that higher energy photons are converted into lower energy photons (Stokes loss). For our system we calculate this ratio to be 0.78 . Figure 2 shows the predicted white power efficiency 
determined by multiplying the measured blue power efficiency data by this ratio. Note that the measured white data disagrees substantially with this prediction as the measured white to blue efficiency ratio is actually greater than one.

We attribute the unpredicted increase in white device efficiency to an increase in light extraction efficiency caused by the light scattering in the Y(Gd)AG:Ce layer at the top surface of the device. To confirm this assignment, a tape of non-absorbing scattering particles in silicone was made and applied to a blue OLED device. Care was taken to use particles with similar size and loading as used in the $\mathrm{Y}(\mathrm{Gd}) \mathrm{AG}$ :Ce tape of the white devices. The resulting device showed a $27 \%$ enhancement in the measured external quantum efficiency relative to the device without the scattering tape. It has been known for many years that texturing the substrate surface can increase external efficiency by providing multiple opportunities for a photon previously not in the escape cone to change angles and exit from a high index medium into the air $^{7}$. We postulate that the same effect is occurring in our devices where the function of the textured surface is achieved by the scattering particles.

In summary, we have demonstrated a down-conversion method that can achieve illumination quality white from OLEDs and should be readily amenable to the type of high volume, low cost processing necessary for lighting applications. It also suggests that, in order to enable lighting applications, research effort should be directed to increasing the efficiency and lifetime performance of the underlying blue OLED. 


\section{Improving Efficiency}

\section{III.1 Theory of Efficiency Enhancement Through Scattering ${ }^{8}$}

In this section, we develop an analytical model to understand the influence of volumetric light scattering on OLED light output and use this to develop design guidelines to maximize device efficiency. In particular, we explore how the amount of light extracted from the OLED depends upon such factors as the particle size, loading fraction and optical loss in the system. In section III.2, we describe detailed experimental measurements that prove the quantitative validity of this model ${ }^{9}$.

It is well known that electroluminescent devices are limited by the optical extraction efficiency, $\eta_{\text {ex }}$, the ratio of light generated within the device to light emitted into the ambient. For OLEDs, typical estimates for this efficiency range between 0.17$0.5^{10,11}$. Thus, a significant fraction of the electrically generated light is lost within the device. This is in sharp relief of mercury vapor fluorescent lighting where the extraction efficiency is close to unity. The loss mechanism in OLEDs arises from light incident at angles above the critical angle of a substrate-air interface that undergoes total internal reflection and is never emitted into the ambient.

For an OLED, the optical extraction efficiency can be divided into two components: the efficiency of light coupling from the active layers into the substrate $\eta_{\text {OLED-s, }}$, and the extraction efficiency from the substrate to the ambient $\eta_{s-a}$ i.e.

$$
\eta_{e x}=\eta_{O L E D-s} \eta_{s-a}
$$

In this section, we focus primarily on the latter term, and in particular we examine the effects of volumetric light scattering upon the fraction of light emitted into the ambient, $\eta_{\mathrm{s}-\mathrm{a}} \quad$ Our discussion will begin with a brief review of the existing approaches to increasing the light output from OLED devices, and some of the peculiarities of the typical OLED geometry that influence the choice of these approaches. This review is not intended to be complete, but rather to frame our discussion of volumetric light scattering.

A number of solutions have been proposed to improve the light extraction, and hence electrical to optical conversion efficiency, from electroluminescent devices ${ }^{12}$. One solution, most applicable to point sources, is to use a shaped substrate, and it was demonstrated that certain lens like shapes will result in almost complete light extraction $^{13}$. This method has been applied to patterned OLED devices as well, where the active area of the OLED was placed at the center of a large hemisphere ${ }^{14}$ or at the bottom of an etched cone. ${ }^{15}$ An alternative approach is to modify the angular dependence of the emission profile of the light emitting source so that most of it is not internally reflected. This control of the angular emission is achieved by placing the emitting layer within an optical microcavity designed to ensure that light is emitted in the forward direction at angles below the critical angle of the interface ${ }^{16,17,18} . \Lambda$ variant of this solution is to place a very low index aerogel ( $\mathrm{n} \sim 1.01)$ next to the thin cavity. If care is taken to ensure that the internal modes of the cavity are suppressed then most of the light 
is coupled into the aerogel layer and ultimately into the ambient due to the small aerogelair index mismatch ${ }^{19}$. Finally, random ${ }^{20,21}$ or structured ${ }^{22,23}$ surface modification of the top output surface of the device can be used to increase the total light output. Here, light scattering at the top surface of the device combined with a reflective bottom surface can result in a net increase in the fraction of light incident at the top surface at angles below the critical escape angle. A photonic surface texture with feature sizes of the order of the wavelength of light ${ }^{24,25,26}$, has also been shown to increase light output from OLEDs however, this approach is more difficult to fabricate.

In this section we focus on modeling the effect of volumetric scattering on the total light output from an OLED device. In principle, volumetric scattering should have the same benefits as surface random surface texturing, but with a very fine level of control. The volume loading of particulate scatterers controls the total amount of scattering, while the average angular deviation induced by each scattering event is controlled through the control of particle shape or size. Volumetric light scattering may be readily introduced through the application of a scattering medium atop an existing OLED device. In the next section we present a detailed development of a volumetric scattering model appropriate for OLEDs. In our application of the radiative transport theory, every attempt was made to limit the number of input parameters and to ensure that these parameters were amenable to experimental determination. In the third section key results from the numerical model of relevance to OLED design are presented.

\section{III.1.A Theory}

In order to develop an analytical approach that can quantitatively describe the effect of volume scattering on the output of an OLED, we employ a radiative transport model. Such models have been developed extensively to describe scattering in different contexts and we take advantage of the extensive work in this topic ${ }^{27}$. In the theoretical development that follows, we first define the OLED and scattering geometry for modeling, then review and utilize the relevant results from single particle scattering theory and the radiative transport approach to multiple scattering. We then build a model for an OLED which incorporates volume scattering. A method for applying this model to calculate the outcoupling efficiency of an OLED in terms of experimentally accessible parameters is then described. We conclude this section with an enumeration of the different approximations that are used in our description of light scattering in OLEDs.

\section{III.1.A.1 OLED and Scattering Geometry}

An actual OLED device consists of several functional layers. In a standard OLED device (Figure 3, top) the bottom most layer seals the device from attack by the ambient. Next is the reflective electrode, organic and transparent electrode layers that form the light emitting structure. Finally, the device is fabricated on a transparent substrate, through which the electrically generated light is coupled into the ambient. To this standard design, we add a layer between the substrate and the ambient consisting of high index scattering particles embedded within a lower index matrix material.

In order to model an OLED device, we approximate the actual, finite, multilayer OLED geometry with a single layer, infinite plane geometry. (Figure 3, bottom). In this 
approximate geometry, the light output can be completely described by 7 parameters, which are shown in bold in the figure. The electrically active layers (cathode, organic, anode) form one boundary of the layer, and their properties determine the reflectance at the bottom interface (at $x=0$ ) and the angular profile of the input light distribution flux within the substrate, $D(\theta)$. For simplicity, the index of refraction of the matrix material of the volume scattering layer and that of the substrate are assumed to be the same. This allows them to be treated as a single effective scattering layer characterized by the scattering and absorption properties of the layer. We characterize this layer using five parameters: $\mathrm{s}$, a, and $\mathrm{g}$, the optical index $\mathrm{n}$ of the substrate, and physical thickness, $\mathrm{X}$. In the body of the text we will discuss and define each of these parameters. The top boundary condition (at $\mathrm{x}=\mathrm{X}$ ), for a flat air-substrate interface, is determined by the Fresnel equation for a medium of optical index $n$.

\section{III.1.A.2 Single Particle Scattering}

The physics of individual scattering events is well understood, and for certain shapes, such as spheres, can be derived exactly using Mie theory ${ }^{28,29}$. From single particle light scattering calculations, there are three quantities of interest: the scattering cross-section, the absorptive loss and the phase function. In a macroscopic sample, the first two quantities are combined with the particle concentration to give a mean free path for scattering, $\mathrm{s}$, and for absorption, $\mathrm{k}$. The phase function is the relative intensity I' of a scattered wave at angle $\left(\psi^{\prime}, \varphi^{\prime}\right)$ with polarization $\pi^{\prime}$, given an incident wave of intensity I at angle $(\psi, \varphi)$, with polarization $\pi$, i.e:

$$
I_{\pi}^{\prime}=p\left(\psi, \varphi, \pi, \psi^{\prime}, \varphi^{\prime}, \pi^{\prime}\right) I_{\pi}
$$

We write the phase function as $\mathrm{p}\left(\psi, \varphi, \pi, \psi^{\prime}, \varphi^{\prime}, \pi^{\prime}\right)$. In our modeling we will introduce several simplifications. We will ignore polarization effects and thus remove any dependence upon $\pi$ or $\pi^{\prime}$. We will also model each scattering event as azimuthally symmetric and thus removing the dependence upon $\phi, \phi^{\prime}$. These assumptions amount to approximating the scattering particles as roughly spherical with randomly oriented facets. This description has been previously used to model phosphor coatings in fluorescent lamps ${ }^{30}$. The remaining angular dependence is only in the difference between the input and output angles, thus we may write the phase function in terms of a single angular variable $\alpha=\psi-\psi^{\prime}$. It is often helpful to express this simplified phase function in terms of the Legendre polynomials $P_{1}$ :

$$
p(\cos (\alpha))=\sum_{l=0}^{\infty} a_{l} P_{l}((\cos (\alpha))) \approx \sum_{l=0}^{L} a_{l} P_{l}((\cos (\alpha)))
$$

We truncate the infinite series at some finite value of $\mathrm{L}$, which can be set very high (i.e. $>50$ ). In principle, the truncation of the series after a finite $\mathrm{L}$ sets a limit on the angular resolution of the model $^{31}$, however, we are always able to find a value of $\mathrm{L}$ sufficiently high so as not to affect the numerical results of interest in this text. If we set $a_{0}=1$, then the phase function will be normalized so that: 


$$
4 \pi=\int p(\cos (\alpha)) d \omega
$$

where the integration is over all solid angles.

While the calculation of the phase function for simple shapes is readily done, the exact calculation of the angular dependence of light scattering from most non-simple particle geometries poses considerable numerical difficulties ${ }^{32}$. To model the broad size distribution of irregularly shaped particles in a typical scattering particle system, we choose a simple form, known as the Henyey-Greenstein (H-G) phase function ${ }^{33}$. This phase function is widely used, and is readily expanded in terms of Legendre polynomials. The expansion terms for the series are:

$$
a_{l}=g^{l}(2 l+1)
$$

where $g$ is the asymmetry of the phase function and is given by the expectation value of $\cos (\psi)$ :

$$
g=\int p(\cos (\psi)) \cos (\psi) d \omega / \int p(\cos (\psi)) d \omega
$$

Thus the detailed physics of light scattering from irregularly shaped particles is replaced by a single parameter that ranges from -1 to 1 . A value of $g=1$ implies that the scattered radiation is not deviated from its original trajectory, whereas a value of $g=-1$ implies that it is directly backscattered. The value of this parameter may be experimentally determined or calculated from first principles. A simple expression that relates $\mathrm{g}$ in the H$\mathrm{G}$ model to physical observables is ${ }^{28}$ :

$$
g=\frac{Q_{e x t}-Q_{p r}}{Q_{s c a}}
$$

where the efficiency factors for radiation pressure $Q_{p r}$, extinction $Q_{e x}$, and scattering $Q_{\text {sca }}$ are computed from a physical theory, e.g. Mie model. For example, a spherical scattering particle with an index of refraction of 1.85 , embedded in a medium of index 1.41, and having radius of $2 \mu \mathrm{m}$, has a value for $\mathrm{g} \sim 0.8-0.85$. Averaging over spherical resonances, this $\mathrm{g}$ tends to increase very slowly as the size is increased ${ }^{27}$. The typical size of commonly available phosphor and scattering pigments ranges from 0.1 to $30 \mu \mathrm{m}$; and thus, one expects to find similar values for $\mathrm{g}$ in many binder/particle systems. A known problem with the H-G phase function is that it leads to non-physical results for very small (relative to the wavelength of light) particles and for these systems use of the Mie theory or alternative parameterization schemes would be preferred ${ }^{34}$.

\section{III.1.A.3. Radiative Transport Approach to Multiple Scattering}

Given a form for the phase function, we need to connect the physics of each scattering event, which can be described either exactly or via approximation, to the macroscopic observables and boundary conditions in a turbid media where a single 
incident photon has undergone several scattering events before leaving the medium. This is the radiative transfer problem and its elucidation in scattering media has been a problem of considerable interest in a number of fields, in particular, astronomy ${ }^{27}$. For an infinite plane-parallel scattering medium, this equation is:

$$
-\mu \frac{d I(x, \mu, \varphi)}{d x}=(k+s) I(x, \mu, \varphi)-\frac{s}{4 \pi} \int_{0}^{2 \pi} \int_{-1}^{1} p\left(\mu, \varphi, \mu^{\prime}, \varphi^{\prime}\right) I\left(x, \mu^{\prime}, \varphi^{\prime}\right) d \varphi^{\prime} d \mu^{\prime}
$$

where $\mathrm{I}(\mathrm{x}, \mu, \varphi)$ is the intensity as a function of position and angle. The variables $\mathrm{k}$ and $\mathrm{s}$ correspond to the mean free path between absorption and scattering events respectively. We have made the standard substitution $\mu=\cos (\theta)$ and it should be noted that the angles $\theta$, and $\varphi$ are in the laboratory frame. We connect these laboratory frame angles with relative angle in the particle frame, $\alpha$, by using the Legendre angle addition theorem:

$$
P_{l}(\cos (\alpha))=P_{l}(\mu) P_{l}\left(\mu^{\prime}\right)+2 \sum_{m=1}^{l} \frac{(l-m) !}{(l+m) !} P_{l}^{m}(\mu) P_{l}^{m}\left(\mu^{\prime}\right) \cos \left(m\left(\varphi^{\prime}-\varphi\right)\right)
$$

Since we will avoid deviating from azimuthal symmetry in either the boundary or the initial conditions, we are able to perform the integral over $\varphi^{\prime}$ to find:

$$
-\mu \frac{d I(x, \mu)}{d x}=(k+s) I(x, \mu)-\frac{s}{2} \int_{-1}^{1} \sum_{l=0}^{L} a_{l} P_{l}(\mu) P\left(\mu^{\prime}\right) I\left(x, \mu^{\prime}\right) d \mu^{\prime}
$$

Several of the different methods that have been developed to solve this problem are summarized in ref. 27. We use the discrete ordinate (DO) approximation which is well described in the literature ${ }^{35,36,37}$ and we will only present a brief summary below.

Within the discrete ordinate approximation, the angle coordinate is discretized, into a set of $\mathrm{N}$ channels, each with index $\mathrm{i}$, and the integral is replaced by a sum:

$$
-\left\langle\mu_{i}\right\rangle \frac{d I_{i}(x)}{d x}=(k+s) I_{i}(x)-\sum_{j}^{N} \frac{\omega_{i} s}{4 \pi} \sum_{l=0}^{L} a_{l} P_{l}\left(\mu_{i}\right) P\left(\mu_{j}\right) I_{j}(x)
$$

where $\left\langle\mu_{i}\right\rangle$ is the average value of $\mu$ in each channel and the additional factor $\omega_{i} / 2 \pi$ arises from normalizing the area of each channel. It is more convenient to work in terms of flux rather than intensity (Following ref 36). The flux in each channel $i$ is related to the intensity by:

$$
F_{i}=\int_{\theta_{i-1}}^{\theta_{i}} I(\theta, \varphi) \cos (\theta) \frac{d \omega}{2 \pi} \approx\left\langle\mu_{i}\right\rangle I_{i}
$$


where $F_{i}$, is the rate of energy flow in a pencil of radiation in an element of solid angle $\mathrm{d} \omega_{\mathrm{l}}$, passing through an element of area da parallel to the boundaries. The equation for the flux as it propagates through the medium now reads:

$$
-\frac{d F_{i}(x)}{d x}=\frac{(k+s)}{\left\langle\mu_{i}\right\rangle} F_{i}(x)-\sum_{j}^{N} \frac{\omega_{i} s}{4 \pi\left\langle\mu_{j}\right\rangle} \sum_{l=0}^{L} a_{l} P_{l}\left(\mu_{i}\right) P\left(\mu_{j}\right) F_{j}(x)
$$

If we then substitute our approximation for the phase function (eq. 4), we arrive at the following coupled set of linear differential equations.

$$
-\frac{d F_{i}(x)}{d x}=\frac{(k+s)}{\left\langle\mu_{i}\right\rangle} F_{i}(x)-\sum_{j}^{N} \frac{\omega_{i} s}{4 \pi\left\langle\mu_{j}\right\rangle} \sum_{l=0}^{L} g^{l}(2 l+1) P_{l}\left(\mu_{i}\right) P\left(\mu_{j}\right) F_{j}(x)
$$

The advantage of this approach is the reformulation of the general integro-differential radiative transfer equation (eq. 7) into this simpler set of coupled equations. The numerical accuracy and angular resolution of the numerical model depends upon the number of channels used. We find that for the accuracy we need for our total flux calculations $(\sim 1 \%)$, between 25 and 60 channels is more than sufficient and leads to matrices whose eigenvalues are readily determined by conventional numerical packages in a sufficiently stable manner.

The boundary conditions are the bi-directional reflectance at $\mathrm{x}=0$ and $\mathrm{x}=\mathrm{X}$, where $X$ is the thickness of the scattering medium. We denote these quantities as $R^{0}\left(\theta, \theta^{\prime}\right)$ and $\mathrm{R}^{\mathrm{X}}\left(\theta, \theta^{\prime}\right)$. These conditions are quite general and can describe either scattering or nonscattering interfaces. We shall make the following simplifications: the boundaries are flat, only specular reflection occurs. Furthermore, the reflectivity of the boundary at $\mathrm{x}=$ 0 is angle independent, and thus described by a single effective cathode reflectivity $\mathrm{R}_{\mathrm{OLED}}$. The reflectance at the top boundary, $\mathrm{R}^{\mathrm{X}}$, is given by the Fresnel equation and is completely characterized by the substrate index $\mathrm{n}$. Within the DO model, these boundary conditions are described by matrices $R_{i, j}^{0}$ and $R^{X}{ }_{i, j}$. In this notation, the off-diagonal elements are the amount of flux reflected from channel $j$ to channel $i$ at each boundary $(0$ or $\mathrm{X}$ ) and the diagonal elements, $\mathrm{R}_{\mathrm{i}, \mathrm{i}}^{0}, \mathrm{R}_{\mathrm{i}, \mathrm{i}}^{\mathrm{X}}$ represent the fraction of the flux that is transmitted through or into the boundary layer.

We are also able to introduce a further simplification that arises from our use of an infinite plane geometry. Formally, the layer thickness, $\mathrm{X}$, is also a required input parameter, however, since the transfer equation depends linearly upon the values of $s$ and $\mathrm{k}$, we can rescale the problem to eliminate the variable $\mathrm{X}$. We do this by substituting defining a new variable $x^{\prime}=x / X$, and define the total scatterance $S=s \cdot X$ and total absorbance $K=k \cdot X$. We then substitute the values of $x^{\prime}, S, K$ for $x, s$ and $k$ in Eqn. 18, and set the top boundary at $X^{\prime}=1$.

\section{III.1.A.4. Calculation of OLED Outcoupling Efficiency}


The input light is assumed to enter the medium at the point $x=0$,and the initial condition is the angular distribution of the light as it emerges from the active layers of the OLED and enters the substrate, i.e. $\mathrm{I}_{0}(\theta)$. We write this condition in terms of flux, $D(\theta)$, and parameterize it by a single number $\mathrm{z}$ :

$$
D(\theta)=\cos (\theta)^{z}
$$

when discretized this condition can be written $D_{i}=\left\langle\mu_{i}\right\rangle^{z}$. The commonly observed Lambertian like emission pattern corresponds to a value of $z=1$. Higher values of $z$ correspond to a more forward directed emission. Such emission patterns are possible if optical microcavity structures are built into the OLED device design ${ }^{26}$. Due to the underlying linearity of the system of equations, this treatment of the initial conditions is quite general, as a given experimental or theoretical angular emission pattern may be represented by a series of orthogonal polynomials in $\mu$. Thus, for a given set of scattering and boundary conditions, we can compute the output coupling for each value of $z$, fit the angular emission profile to a polynomial expansion, and take the appropriate weighted sum.

Given the initial and boundary conditions we solve Eqn. 18 to find the amount of flux $\mathrm{F}$ that 'leaks' from each angular channel to the ambient.

$$
F_{i}^{\text {out }}=\left(R_{i, i}^{X}\right) F_{i}(X)
$$

where $R_{i i}{ }^{X}$ is equal to the transmittance across the interface for each angular channel $i$, for planar interfaces it is determined by the Fresnel equation. The fraction of light outcoupled from the substrate to the air can be expressed as:

$$
\eta_{s-a}=\frac{\sum F_{i}^{o u t}}{\sum D_{i}}
$$

Note that, in the absence of scattering, $F_{i}(X)=D_{i}$, and we recover the usual result that the light output is determined by the optical index and the shape of the input light distribution. In this case the substrate to air coupling efficiency is well approximated by:

$$
\eta_{s-a}=\int_{0}^{\theta_{c}} D(\theta) \sin (\theta) d \theta
$$

where $\theta_{c}=\operatorname{asin}(1 / n)$, the critical angle. If one combines this result for $\eta_{\mathrm{s}-\mathrm{a}}$ with an appropriate microcavity model for $\eta_{\text {oled-s }}$ in eq. 1 , one recovers the usual estimates for the total out-coupling efficiency $\eta_{\mathrm{ex}}{ }^{38}$.

\subsection{A.5. Summary of Approximations}

In the course of our analysis, we have made several approximations that may be grouped into eight categories. We have assumed that: 1) The scattering layer and OLED 
substrate can be modeled as infinite homogeneous, isotropic layers; 2) scattering events and the radiation source possess azimuthal symmetry; 3) polarization effects are relatively unimportant; 4) a single approximate phase function models single scattering events over all wavelengths, and there is no interference between scattering events; 5) We can use an average effective cathode reflectivity that is independent of angle and position; 6) the top surface is flat; 7) we can ignore fluorescence effects. The eighth category results from errors introduced by our particular choice of numerical solution which are discussed in the literature ${ }^{36,37}$, and they will not be addressed further here.

The first set of approximations, which maps the problem to the realm of atmospheric physics, is probably the most significant. Clearly, an infinite layer approximation is suspect if the OLED is on a very small substrate where emission from the sides is a significant fraction of the total emitted light. However, for applications such as lighting, there is a trend towards using thinner, larger area OLEDs with a high device fill factor. In this case, the amount of light emitted from the sides of the substrate is small, and the infinite layer approximation should be acceptable. For the thin, transparent substrates typical for OLEDs, the medium is well modeled by single layer. However, if required, this assumption is readily relaxed by treating the system as a succession of homogenous layers ${ }^{37}$, which amounts to using the model separately on each layer. The requirement that each layer be homogeneous is equivalent to assuming that one is always interested in behavior with length scales much greater than those of a single particle or average particle separation. Since these distances are usually on the order of microns, and the substrates are usually on the order of millimeters in thickness, this approximation is also well justified.

The remaining approximations simplify the radiative transfer equation significantly. The second approximation is justifiable on an empirical basis. It adequately describes light scattering from many phosphor systems and the radiation pattern from typical OLED devices, although some OLED devices in which the emission pattern or scattering is dominated by grating effects will not be readily described using this approximation. The third approximation leads to the largest errors in the case of small amounts of light scattering ${ }^{36}$. Indeed, polarization effects are seen in the OLED emission pattern of standard flat substrates ${ }^{11}$. In terms of our model, this implies that one polarization is characterized by a different value of $z$ in the expression $D(\theta)=\cos (\theta)^{z}$. However, as will be shown in section III.1.B, changes in $\mathrm{z}$ lead to relatively small effects in the presence of strong light scattering, and thus in many cases, polarization effects can be ignored.

The fourth and fifth approximations group many different physical effects together into single parameters. The fourth approximation groups the details of the physics of single particle light scattering into the parameter g. Given that we are only interested in the total flux emerging from the device, and not any angular details, results from the atmospheric scattering literature suggest that this parameterization will not induce large errors ${ }^{34}$. The fifth approximation groups the reflectance of the metal cathode and thin film OLED layers into a single average number, ROLED, which represents the angle and polarization averaged specular reflectance. Calculation of the polarization 
averaged, angle dependent reflectivity for an idealized aluminum-glass $(\mathrm{n}=1.5)$ interface revels that it varies between 0.87 and 0.84 in the range between 0 and 80 degrees. Given this minor variation, our approximation should be adequate. If the cathode is textured in some manner, then a non-specular component to the reflectance is required.

The assumption that the top surface is flat may be relaxed, if necessary, through the introduction of non-specular reflection terms in the top boundary condition. There are several top-surface texturing schemes in the literature that are known to improve light output $^{15,19}$; these may be approximated by non-specular reflection coefficients in the top boundary. The required new inputs in this case are the Bi-Directional Reflectance Function (BDRF) of each surface, which can be readily measured. For purposes of the model, any texturing or emission pattern is readily parameterized, provided that the condition of azimuthal symmetry is maintained.

Fluorescent or phosphorescent scattering systems may also be handled by the inclusion of additional equations that describe the flux in each channel, but at a different wavelength. This works out to doubling the number of channels so that the downconverted wavelength has its own gain and loss terms. Using this extension of the model coupled to a microcavity model of light generation within the OLED would allow for a complete description of our demonstrated white light OLED.

\section{III.1.B Results}

In the previous section we provided a detailed development of a 7-parameter model of radiative transport appropriate for OLED devices. In this section we discuss some the implications of this model for light extraction. The model is sufficiently flexible to describe most large area flat OLEDs and at the same time all of the parameters are accessible through experimental measurement. In a companion paper, we present a set of experiments that prove the validity of this model for real OLED systems. In this section we map our model parameters to OLED design parameters and discuss the impact of these choices upon light extraction.

\subsection{B.1. Relation between model parameters and device design variables.}

Within the context of the model, the OLED device design sets three parameters the index of refraction of the substrate (n), the effective reflectivity of the bottom layer, $\mathrm{R}$, and the angular distribution of the emitted light as it enters the substrate from the active layers of the OLED, $\mathrm{D}(\theta)$, which is characterized by the parameter $\mathrm{z}$. These variables can be directly measured and are controlled through choices in device design. For example the reflectance parameter includes not only the losses associated with the metal film, but also absorption losses incurred by the transparent electrode and organic layers. The presence of these additional layers limits the reflectance to a lower value than the theoretical maximum dictated by the metal and substrate complex dielectric constants. The impact of multiple layer OLED structures on the angular light distribution is well known, and through the use of multiple layers and narrow ranges of emission wavelengths, the light output can be highly directed, thus altering $\mathrm{z}$. For a typical 
OLED fabricated on glass with weak microcavity effects, typical values for these three parameters are $\mathrm{R}_{\mathrm{OLED}}=0.8, \mathrm{n}=1.5$, and $\mathrm{z} \sim 1-2$.

The remaining parameters in the model relate to the volume scattering medium. In general, absorptive losses for scattering systems can be made very low i.e. $\mathrm{k}<$ 0.001.Thus, in the remainder of the discussion absorption loss in the scattering medium will be ignored. The total scatterance, $\mathrm{S}=\mathrm{s} \mathrm{X}$ is controlled by the mean free path between scattering events, $\mathbf{s}$ and the substrate thickness, $X$. Experimentally, the scatterance may be adjusted through a combination of the scattering particle loading and scattering layer thickness. Thus, the parameter, $\mathrm{S}$, is broadly tunable but for most applications varies in the range 0 to 6 . The asymmetry parameter, $g$, is controlled by the size, shape, and the relative optical index of the scattering particles.

\section{III.1.B.2 Design Configurations to Maximize Light Extraction}

If we solve the model with the typical values for the OLED parameters described above and set $\mathrm{S}$ to zero to describe the typical case with no volume scattering, we get a value for the light extraction term, $\eta_{\mathrm{s}-\mathrm{a}}$ of 0.44 which is in agreement with the usual estimates (Eq. 18) ${ }^{38}$. From this, one can then calculate the total extraction efficiency, $\eta_{\mathrm{ex}}$, if the extraction efficiency from the active layers of the device into the OLED substrate, i.e. $\eta_{\text {OLED-s, }}$ is known. Estimates of $\eta_{\text {OLED-s }}$ can be calculated using the microcavity models developed in the literature. $A$ simple estimate is obtained by ignoring detailed microcavity effects and assuming that $\eta_{\text {OLED-s, }}$, can be modeled by the Fresnel eqn. i.e. $\eta_{\text {OLED-s }}=\left(n_{\text {substrate }} / \mathrm{n} \text { OLED }\right)^{2}$, and assuming a Lambertian emission profile within the OLED layer. For example, setting the index of the glass substrate, $n$, to 1.5 and OLED active index to 1.7 , then $\eta_{\text {OLED-s }}=(1.5 / 1.7)^{2}=0.78$. If we substitute this simple estimate along with our model results, we predict an output coupling efficiency without volume scattering of $(0.75)^{*} 0.44=0.33$, similar to the results obtained by Kim et. al. for polymeric OLED's ${ }^{10}$.

In the following, we show the results of model calculations of $\eta_{s-a}$ with volume scattering included. We then use these to discuss the tradeoffs between design parameters and overall output extraction efficiency.

\section{III.1.B.2.A OLED Reflectivity}

We find that in the presence of light scattering the most critical parameter in the determination of light extraction is the effective cathode reflectivity. In figure 4, extraction efficiency is plotted versus scatterance for different values of the effective cathode reflectivity. Note that for the chosen range of effective reflectivity values, there is a large range of scatterance values where the substrate-air extraction efficiency is enhanced relative to the zero scatterance value (0.44). As the effective reflectivity decreases, the maximum enhancement also decreases. Although not depicted in the figure, we find that enhancement still occurs as long as the effective reflectivity is greater than 0.2 . For high cathode reflectivity, $\eta_{\mathrm{s}-\mathrm{a}}$ can be nearly unity i.e. very efficient "photon recycling" occurs. Intuitively, this is expected since, in the absence of losses a given photon can impinge upon the interface many times until in escapes. For a given reflectivity, the overall shape of the curve as a function of scatterance is also readily 
explained. At low values of scatterance, waveguiding within the substrate is not completely suppressed, while at very high values of scatterance, the light is mostly reflected from the substrate back to the lossy cathode. The peak value is the point where these two effects are balanced. Note that for a typical OLED reflectivity of 0.8 , the extraction efficiency is enhanced by approximately a factor of 1.6 from the zero scatterance value. Thus the total output coupling efficiency is increased from $\sim 0.3$ to $\sim 0.6$.

\section{III.1.B.2.B. Scattering Particle Shape and Size}

In our model, the effects of the particle shape and size are contained within a single parameter, g. In Figure 5, we plot $\eta_{\mathrm{s}-\mathrm{a}}$ vs. scatterance for four different values of $\mathrm{g}$ ranging from $\mathrm{g}=0$ to $\mathrm{g}=0.9$. The emission profile was assumed to be lambertian $(\mathrm{z}=1)$, and the reflectance of the cathode was set at 0.8 . Note that for each value of $g$, there is a peak in extraction efficiency at a particular scatterance value as expected from figure 4. However, as the value of $\mathrm{g}$ is increased the peak position is shifted to higher values of the scatterance. Physically, it is possible to understand the reason for this shift. The parameter $\mathrm{g}$ represents how much, on average, the scattered light is deviated from its original direction. Thus particles that are characterized by values of $g$ near 1 do not efficiently deflect the incident beam, and at low loadings, should exhibit extraction efficiencies similar to non-scattering substrates. In this case, optimal light extraction will occur only at very large values of scatterance. On the other hand, low values of $g$ imply better scattering efficiency and hence less scatterance is required to achieve optimal scattering. Thus, as $g$ is varied between 0 and 1 , one expects that the optimal value of $S$ for light extraction should be shifted to higher values. Ultimately, in the limit where $\mathrm{g}$ goes to 1, infinite scatterance would be required to achieve optimal light extraction.

It should be noted that as $\mathrm{g}$ increases, the value of the peak efficiency also increases. However, this increase in peak value is relatively small, from 0.71 to 0.74 . Thus, it seems that, the maximum attainable extraction efficiency is nearly independent of $\mathrm{g}-$ and hence independent of scattering particle size and shape. However, the falloff of $\eta_{\mathrm{s}-\mathrm{a}}$ as $\mathrm{S}$ increases becomes more gradual as $\mathrm{g}$ is increased, implying a much wider operating range for the loading fraction of particles that exhibit higher values of $g$. Thus, to maximize design flexibility and tolerance, larger grained scattering particles that exhibit higher value of $g$ are preferred.

\section{III.1.B.2.C Substrate Index of Refraction}

In the absence of scattering, the value of $\eta_{\mathrm{s}-\mathrm{a}}$ is determined by the index of refraction in the substrate. To test if this remains true in the presence of light scattering, we plot the calculated extraction efficiency as a function of cathode reflectance for different values of substrate index. (Figure 6) The scatterance in each case was set to a value that maximized light extraction. As expected, the required scatterance was nonzero for all substrate indices except for $n=1$. Consistent with the discussion above, for all values of $n$ greater than 1 , the extraction efficiency increases to the maximum possible value of unity as the effective cathode reflectance increases towards unity. However, for lower values of cathode reflectance, the optical index of the substrate sets an upper limit 
on the extraction efficiency, $\eta_{\mathrm{s}-\mathrm{a}}$ which increases as the substrate index decreases. Thus, optimization of $\eta_{\mathrm{s}-\mathrm{a}}$ requires the use of low index substrates.

Changing the substrate index also affects $\eta_{\text {oled-s. }}$ In fact, if one ignores optical microcavity effects, maximization of $\eta_{\text {oled-s }}$, ideally requires a substrate index matched to that of the active organic layers $(n \sim 1.7)$ to eliminate Fresnel reflection and waveguiding within the active layers. This leads to the following question: To maximize total extraction efficiency, is it better to use a low index substrate, and maximize $\eta_{\mathrm{s}-\mathrm{a}}$ at the expense of $\eta_{\text {oled-s }}$ or is it better to use a high index substrate and maximize $\eta_{\text {oled-s }}$ at the expense of $\eta_{\mathrm{s}-\mathrm{a}}$ ? In the OLED literature, it has been shown that increasing the substrate index and texturing the top surface to increase $\eta_{\mathrm{s}-\mathrm{a}}$ increases OLED output ${ }^{22}$ and that reducing the effective optical index of the substrate also increases light output ${ }^{19}$. Which approach is best from the point of view of maximizing the overall extraction efficiency, $\eta_{\mathrm{ex}},(\mathrm{Eq} 5)$, requires an analysis of the microcavity structure, which affects both $\eta_{\text {OLED-s }}$ and $\eta_{\mathrm{s}-\mathrm{a}}$ through angular emission modification (the $\mathrm{z}$ parameter in our model). Depending upon the exact OLED structure it is possible to obtain improvements via both routes. While detailed design optimization of the combined microcavity and scattering is beyond the scope of our present discussion, our model does allow us to illustrate some of the possible tradeoffs between increased light scattering and microcavity optimization on $\eta_{\mathrm{s}-\mathrm{a}}$; we explore these in the next section.

\section{III.1.B.2.D Influence of the Optical Microcavity}

The effect of the optical microcavity structure of an OLED on $\eta_{\mathrm{s}-\mathrm{a}}$ can be taken into account in this model by modifying $z$, which determines $D(\theta)$. The inset to figure 7 shows the light distribution as a function of angle for different values of $\mathbf{z}$ (solid lines). For comparison, we show a complete optical microcavity calculation of the angular emission from a typical polymer OLED device structure. Specifically, we show the calculated polarization averaged angular emission profile using the method described by Crawford $^{39}$, which is similar to the other detailed models presented in the literature ${ }^{10,11}$. We have assumed that the emission occurs from a well-defined plane in the device, with either dipoles aligned parallel to the electrodes or isotropically oriented. It should be noted that while the output of the microcavity calculations depends in detail upon the assumptions used for layer thickness, indices of refraction, and emission, the calculated distributions presented here are representative of the typical emission pattern observed in OLED devices where strong microcavity effects are not specifically engineered into the device structure. For such cases, we have found that in general, values of $\mathrm{z}$ less than 2 provide a reasonable approximation for $\mathrm{D}(\theta)$

In order to ascertain the interplay between optical microcavity effects and scattering enhancement of $\eta_{s-a}$ on the choice of substrate index, model calculations were performed. In these calculations, we maximized $\eta_{\mathrm{s}-\mathrm{a}}$ by optimizing the scatterance for a given substrate index assuming three different input angular distributions, $\mathrm{D}(\theta),-\mathrm{z}=0$ (isotropic distribution), $z=1$ (lambertian distribution), and $z=2$. The anisotropy, $\mathrm{g}$, was set equal to 0.9 . The results are depicted in figure 5 . One can see that, for each $\mathrm{z}$ value, the curves exhibit the same basic behavior as a function of substrate index. This indicates 
that, for this range of $\mathrm{z}$, the choice of substrate index of refraction is not complicated by coupling between microcavity effects and scattering enhancement effects. Strong microcavities can be engineered into an OLED, for instance, with a dielectric stack mirror or using thin metal films. In such cases, much more peaked angular dependence (eg. $\mathrm{z}=8$ ) is possible and thus a strong microcavity has the potential to increase the extraction efficiency by ensuring that most of the light is emitted within the critical angle escape cone ${ }^{16-18}$. It is thus of interest to examine how a strong microcavity interacts with volume scattering. Figure 6 presents the results of a model calculation of $\eta_{\mathrm{s}-\mathrm{a}}$ versus both scatterance and $\mathrm{z}$. For these calculations, a substrate index of 1.5 and an anisotropy value of 0.8 were assumed. The weak microcavity regime typical of most OLEDs corresponds to the area where $\mathrm{z}$ is less than 2 and in this regime one can see that finite scattering ( $>0$ ) is always beneficial in enhancing external $\mathrm{QE}$. The opposite behavior occurs for strong microcavities $(z>6)$ where light extraction efficiency decreases as scattering is introduced. This occurs because scattering diverts some of the highly forward-directed light output away from the critical-angle escape cone. However, this efficiency decrease is relatively gradual as $\mathbf{S}$ is increased. In fact, the data presented in Figure 8 shows that that the difference in $\eta_{\mathrm{s}-\mathrm{a}}$ between using a strong microcavity without scattering (eg. $z=8$ and $S=0$ ) and using a weak microcavity with optimized scattering (eg. $z=2$ and $S \sim 2.5$ ) is only $\sim 0.2$. In addition, it is important to note that the angular emission from a strong microcavity is also highly wavelength dependent. So for a broad light source such as a white light emitting OLED, it is difficult to achieve a high $\mathrm{z}$ value averaged over all emission wavelengths. For such cases, it is likely that a weak microcavity with optimized scattering is an easier and more robust method for achieving external efficiency enhancement. 


\section{III.2 Experimental Demonstration of Efficiency Enhancement Through Scattering ${ }^{9}$}

In the last section, a radiative transport model was developed to provide a description which is at once flexible enough to parameterize and describe these different schemes, and at the same time, capable of quantitative prediction of absolute extraction efficiencies based upon experimentally determinable inputs. In this analysis, many simplifying approximations were made in an effort to minimize and simplify the number of input parameters. The goal of this section is to present a set of experimental measurements of both the model input parameters and the relative extraction efficiency as a function of volumetric light scattering in order to test the model's capability for predicting the extraction efficiency of real devices.

Our goal is made possible by the fact that a light emitting OLED and a scattering film may be separately fabricated, individually characterized and then optically coupled together. We first characterize all the relevant parameters of freestanding, scattering films, using the usual tools of reflectance, transmission and angularly resolved scattering. We then collect optical data on the OLED device, characterizing its reflectance and emission properties, again using standard methods. Given the optical characteristics of the OLED and the film, the radiative transport model predicts the effect on light output when the two pieces are combined, without the inclusion of any additional adjustable parameters. In addition, we can successively attach many different scattering films to the same OLED, and accurately determine how the light output varies as function of different scattering parameters. Thus, the model's ability to predict the dependence of light output as a function of scattering parameters can be tested. These detailed comparisons of both magnitude and functional dependencies provide a stringent test of the radiative transport model.

Our discussion will be organized into two main sections. The first section introduces the critical concepts from the model necessary to understand our interpretation of the experimental data. The discussion of the model differs considerably from the presentation given in Section III. 1 in that we concentrate on providing a qualitative overview and physical picture rather than a detailed development. Section III.2.B contains a detailed exposition of our experimental methodologies and the comparison of the experimental data to model calculations.

\section{III.2.A Model}

In this discussion, it is useful to decompose the total extraction efficiency $\eta_{\mathrm{ex}}$ into two components: i.e.

$$
\eta_{e x}=\eta_{\text {OLED-s}} \eta_{s-a}
$$

where $\eta_{\text {OLED-s }}$ is the fraction of the electrically generated light that is coupled into the substrate, $\eta_{\mathrm{s}-\mathrm{a}}$ is the fraction of light that is coupled into the substrate that is emitted into the ambient. This decomposition is analogous to the usual distinction made between ITO/organic and substrate wave guided modes ${ }^{10}$. In this paper, the latter term is the 
primary focus. In particular, we examine the effects of volumetric light scattering upon the fraction of light emitted into the ambient, $\eta_{\mathrm{s}-\mathrm{a}}$.

The basic physical processes that must be modeled are illustrated schematically in Figure 9. On the left side of the figure is shown an idealized OLED and a scattering, but non-absorbing, layer. In translating the physical structure to a mathematical model, we group the OLED active layers into a single layer and focus primarily on the different physical processes that occur within the scattering layer (Figure 9 right). These processes can be illustrated by following the history of a typical ray from the OLED to the air. Light emerges from the active layers of the OLED with a angular distribution characterized by the function $\mathrm{D}(\theta)$ at position "a". The ray impinges upon a particulate scatterer at "b", and is back scattered to point "c", which is the surface of the active layers of the OLED. The ray is reflected at point "c", but also attenuated due to the finite reflectivity $R_{\text {OLED }}$ of the OLED. The ray then travels to point "d", is scattered again, and reaches the top air-glass interface a point "e" with an angle of incidence that exceeds the critical angle and undergoes total internal reflection. The ray is reflected back to a scattering particle (" $\mathrm{f}$ "), is back-scattered toward the air substrate interface (point " $\mathrm{g}$ "). This time the angle of incidence is less than the critical angle and the ray is transmitted across the interface.

This example highlights the necessary physical parameters needed to model volume scattering in OLEDs. First, the internal angular distribution of light, $\mathrm{D}(\theta)$, as it emerges from the OLED and goes into the substrate must be characterized. Due to optical microcavity effects, $D(\theta)$, is in general not a simple function of angle and is polarization dependent. In many cases, the polarization may be averaged, and the distribution approximated by a simple form:

$$
D(\theta)=\cos (\theta)^{z}
$$

which is characterized by a single parameter, $\mathrm{z}$. Second, the angular distribution of each single particle scattering event must be determined. This distribution is given by the phase function which is expressed as the relationship between the input intensity I, and output intensity, I':

$$
I^{\prime}=p\left(\theta^{\prime}, \theta\right) I
$$

For simplicity, we have assumed azimuthal symmetry and polarization independence,

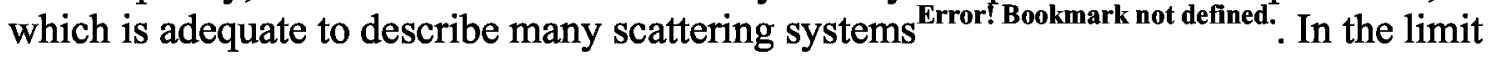
of isotropic scattering, $p\left(\theta^{\prime}, \theta\right)=1$. However, for most micron sized particles, $p\left(\theta^{\prime}, \theta\right)$ is peaked in the forward direction, i.e. where the difference $\theta^{\prime}-\theta$ is small. The form of the phase function can be quite complex, but we shall further simplify our analysis by using a one parameter form of the phase function due to Henyey and Greenstein ${ }^{40}$ :

$$
p_{1 H G}\left(\theta, \theta^{\prime}\right)=\frac{1-g^{2}}{\left(1+g^{2}-2 g \cos \left(\theta-\theta^{\prime}\right)\right)^{3 / 2}}
$$

where $g$, the asymmetry factor, is the expectation value of $\cos \left(\theta-\theta^{\prime}\right)$. Thus, $g=1$ implies that each scattering event does not deflect the beam, $g=-1$ implies that each scattering 
event back scatters the beam along the incident direction, and $\mathrm{g}=0 \mathrm{implies}$ isotropic scattering.

The third necessary physical parameter is the probability that a scattering event will occur as the ray traverses the substrate. This is a product of the scattering crosssection, the particle concentration, and the total substrate thickness. It can be characterized by a single parameter, $\mathrm{S}$, the total scatterance. The fourth necessary input is the effective reflectance of the OLED active layers and cathode. We assume specular, angle independent reflection characterized by a single parameter $\mathrm{R}_{\mathrm{OLED}}$. Finally, the critical angle given by:

$$
\theta_{c}=\sin ^{-1}\left(\frac{1}{n}\right)
$$

must be measured. Here $\mathrm{n}$ represents the index of refraction of the substrate.

The five physical parameters - $\mathrm{z}, \mathrm{g}, \mathrm{S}, \mathrm{R}_{\mathrm{OLED}}$, and $\mathrm{n}$ - determine the extraction efficiency from an OLED device. While the introduction of these parameters has been somewhat heuristic, this is the same parameter set that emerged from a more formal physical analysis in the companion paper. The five parameters can be grouped into two categories, one set - $\mathrm{n}, \mathrm{z}$, and ROLED - is common to all large, planar OLEDs. The other set $-\mathrm{n}, \mathrm{S}$, and $\mathrm{g}$ - is common to all scattering layers. For simplicity, we have assumed a common index of refraction for both the OLED substrate and the scattering layer. This grouping of the model input parameters matches our experimental design of separate measurements of OLED and scattering film optical properties.

The physical picture presented in figure 9 and developed in the previous paragraph can be expressed mathematically through the following integro-differential equation $^{\text {Error! Bookmark not defined.,41. }}$

$$
-\mu \frac{d F\left(x^{\prime}, \mu\right)}{d x^{\prime}}=(K+S) F\left(x^{\prime}, \mu\right)-\frac{S}{2} \int_{-1}^{1} \sum_{l} g^{l} P_{l}(\mu) P_{l}\left(\mu^{\prime}\right) F\left(x^{\prime}, \mu^{\prime}\right) d \mu^{\prime}
$$

The flux, F, for a given wavelength, is defined as the amount of light passing through an area $\mathrm{dA}$, in the plane parallel to the interfaces, in a slice of solid angle $\mathrm{d} \omega . \mathrm{K}$ and $\mathrm{S}$ are the total absorbance and scatterance. For the work described in this paper, $\mathrm{K}$ is nearly 0 . We have made the substitution $\mu_{i}=\left\langle\cos \left(\theta_{i}\right)\right\rangle$, and $P_{1}$ are the Legendre polynomials of order 1. $x^{\prime}$ is the reduced distance into the medium, $x^{\prime}=x / X$, so the boundaries are at $x^{\prime}=$ 0 and $x^{\prime}=1 . \mathrm{g}$ is the anisotropy of each scattering event as defined by Eqn 26 .

With three parameters, Eq. 28 gives the flux at every point in the system and so completely solves the predicted light distribution for plane parallel systems in the presence of scattering. Different experimental geometries are specified through appropriate modification of the boundary and initial conditions. For purposes of this paper, there are two basic experimental geometries - a free-standing scattering film and scattering film coupled with an OLED; we now present these boundary and initial conditions for our two geometries. 


\section{III.2.A.1 Free Standing Film}

For a free-standing film with dispersed scattering particles, the boundary conditions to equation 6 are given by the specular reflection coefficients for each face. These are given by the Fresnel equations. For the upper face at $\mathrm{x}^{\prime}=1$ :

$$
\begin{gathered}
R_{1}(\mu)=\frac{1}{2}\left(R^{\|}(\mu)+R^{\perp}(\mu)\right) \\
R^{\|}(\mu)=\left|\frac{\mu-n^{-1} \beta}{\mu+n^{-1} \beta}\right|^{2} \\
R^{\perp}(\mu)=\left|\frac{\beta-n^{-1} \mu}{\beta+n^{-1} \mu}\right|^{2}
\end{gathered}
$$

At the lower face, $x^{\prime}=0, R_{0}(-\mu)=R_{1}(\mu)$. The subscripts denote the location of the boundary, either at $x^{\prime}=0$ or $x^{\prime}=1$, and $\beta$ is defined as:

$$
\beta=\sqrt{1-n^{2} \sqrt{1-\mu^{2}}}
$$

In the experiments described in section III.2.B, the free standing film is interrogated with a collimated beam of light, In this case the input light distribution at the lower face of the film is given by a delta function:

$$
D(\theta)=\delta(\theta)\left(1-R_{f f}\right)
$$

Where $R_{\mathrm{ff}}$ the reflectance at normal incidence of the air-medium interface. The $\left(1-\mathrm{R}_{\mathrm{ff}}\right)$ term accounts for the light that is reflected from of the film that occurs before it enters the scattering media. Given these conditions and a set of corresponding set of $\mathrm{S}, \mathrm{K}$, and $\mathrm{g}$ values, eq. 28 can be solved to provide the total reflectance, $R$, transmission, $T$, as follows:

$$
\begin{aligned}
& R=\int_{-1}^{0}\left(1-R_{0}(\mu)\right) F(0, \mu) d \mu+R_{f f} \\
& T=\int_{0}^{1}\left(1-R_{1}(\mu)\right) F(1, \mu) d \mu
\end{aligned}
$$

The measured inline transmission, $T_{\text {inline, }}$ is calculated by integrating the flux transported across the film-air interface over the half-angle subtended by the detector, $\theta_{\mathrm{d}}$ :

$$
T_{\text {inline }}=\int_{\cos (\theta t)}^{1}\left(1-R_{1}(\mu)\right) F(1, \mu) d \mu
$$

\section{III.2.A.2 OLED + Scattering Film}

We also examine the case where the film is affixed to an OLED device. The input light distribution is now given by:

$$
D(\theta)=\mu^{z}
$$


where the parameter $z$ describes the angular dependence of the OLED emission and is an experimental observable. The top boundary condition (at the air-film interface) is the same as for the free-standing film, but the bottom boundary condition (the OLED-film) interface is replaced by a specular reflectance condition given by:

$$
R_{0}(\theta)=R_{\text {OLED }}
$$

Using the model we can compute the total flux in each channel that is outcoupled to the ambient, $\Phi^{\text {out }}(\theta)$ as a function of internal angle, $\theta$ :

$$
\Phi^{\text {out }}(\theta)=\left(1-R_{1}(\cos (\theta))\right) F(1, \cos (\theta))
$$

The internal flux at the boundary, $\Phi^{\text {out }}(\theta)$, is related to the observed Flux as a function of angle $\varphi$ outside the medium, $\Phi^{\mathrm{ex}}(\varphi)$ :

$$
\Phi^{e x}(\varphi)=\Phi^{\text {out }}(\theta) \frac{d \Omega_{\text {int }}}{d \Omega_{\text {ex }}}=\Phi^{\text {out }}(\theta) \frac{\cos (\varphi)}{n^{2} \cos (\theta)}
$$

which appears as Eqn 5. in Kim et. al. ${ }^{10}$, where the ratio in the middle and rightmost equations is the ratio of solid angles in each medium. The fraction of light out-coupled from the substrate to the air out of the total amount of light coupled into the substrate can be expressed as:

$$
\eta_{s-a}=\frac{\int \Phi^{o u t}(\theta) \sin (\theta) d \theta}{\int D(\theta) \sin (\theta) d \theta}
$$

\section{III.2.B Experimental:}

In this section, experiments aimed at measuring the relevant scattering parameters from free-standing scattering films are described. In addition, measurements of the relevant physical parameters of an OLED device are described. The resulting parameters are then used to predict the effect on light output when the scattering film is optically coupled to the OLED device without the use of any adjustable parameters

\section{III.2.B.1 Scattering Properties of Freestanding Films}

\section{III.2.B.1.A Film Fabrication}

Freestanding tapes with variable optical scattering were prepared by mixing a known weight of non-visible light absorbing particles with $10 \mathrm{~g}$ of uncured PDMS resin ( $\mathrm{n}=1.41$ for the cured film). The two white powders used in this study were a sample of cool white $(\mathrm{CW})$ phosphor $\left(\mathrm{d}_{50}=6 \mu \mathrm{m}\right)$, and $\mathrm{ZrO}_{2}$ powder $\left(\mathrm{d}_{50}=0.6 \mathrm{~mm}\right)$. The median particle sizes were determined via light scattering. Typical weight loadings ranged between $0.2 \%-1.76 \%$ for the $\mathrm{ZrO}_{2}$, and $1 \%-20 \%$ for the cool white phosphor particles. Infinite plaque reflectivity measurements performed on both samples indicated very low visible light absorbance. The scattering films were prepared by tape casting prior to curing. Typical film thickness was about 400-600 $\mu \mathrm{m}$. 


\section{III.2.B.1.B. Measurement of Scatterance (S)}

To determine the degree of light scattering in the free standing films, it is necessary to use the radiative transport models in reverse, i.e. starting from and observed set of transmission and reflectance data, invert to obtain a set of S, K, and $\mathrm{g}$. In general this procedure requires a non-linear fit of the three inputs $\left(T, R, T_{i n l i n e}\right)$ to the three outputs $(\mathrm{S}, \mathrm{K}, \mathrm{g}$ ), but in the cases where these is very little absorption (i.e. $\mathrm{K} \sim 0$ ); $\mathrm{S}$ is determined from the inline transmission directly via a Beer's law type expression:

$$
S=-\ln \left(T_{\text {inline }}\right)
$$

The measurement of inline transmission consisted of passing a chopped laser beam through a freestanding film. The beam was taken from the $488 \mathrm{~nm}$ line of an Ar ion laser, or from the $670 \mathrm{~nm}$ emission of a power stabilized diode. A $1 \mathrm{~cm}$ diameter diode connected to a preamplifier and then to a lockin amplifier was placed at a variable distance behind the suspended film. The angular resolution of the system was at least $\sim 1^{\circ}$ $\left(\theta_{d}=0.5^{\circ}\right)$ in all cases, care was taken to mount the film so that the unscattered portion of the laser beam was not deflected away from the diode. The measurements were repeated at 3-5 different positions on each tape. The incident intensity of the laser beam on the diode was measured both with and without the sample in position and the two signals ratioed to yield an inline transmission. The measured variation in the calculated scatterance, $\mathrm{S}$ was $<+/-0.07$. A possible systematic error is contamination of the inline transmission signal by low angle light scattering that is stronger than the weak inline component. To guard against this possibility, the inline transmission was measured as a function of distance from the detector, and care was take to ensure that the extracted value of S did not increase as the detector was moved farther away from the sample. For samples with relatively high particle loadings, we determined $\mathrm{S}$ via a linear fit of the scatterance versus the known weight loading. We find that for both our $\mathrm{ZrO}_{2}$ and $\mathrm{CW}$ samples there is very little variation of $\mathrm{S}$ as a function of wavelength, consistent with the predictions obtained from scattering theory for particles in this size regime.

\section{III.2.B.1.C. Measurement of Scattering Anisotropy (g)}

Given $\mathrm{S}, \mathrm{K}$, and $\mathrm{n}$, we can measure $\mathrm{g}$ by fitting equations $34-35$ for transmission and reflectance; $g$ is the only adjustable parameter ${ }^{42}$. The total transmission and reflectance spectra were measured using the integrating sphere accessory attached to a commercial UV-Vis spectrometer (Perkin-Elmer lamda-9). The error at any wavelength point was measured at $+/-0.3 \%$. We find that the for the larger $\mathrm{CW}$ particles there is almost no variation in the total transmission and reflectance values as across the visible range $\left(400 \mathrm{~nm}-700 \mathrm{~nm}\right.$ ), whereas for the $\mathrm{ZrO}_{2}$ loaded tapes, the tapes become slightly more reflective at shorter wavelengths. Given values of $\mathrm{S}$ (determined from our inline measurements), $\mathrm{K}$ (which we set to $<0.001$ ), and $\mathrm{n}$ of the PDMS, and g, Eq. 27 predicts the values of total reflection and total transmittance. For our tapes we fit $\mathrm{g}$ to best match the observed transmission and reflectance values. This procedure is quite robust and our observed standard deviation in the determination of $\mathrm{g}$, pooled over all samples, is 0.008 . A comparison of the observed and calculated values of transmission and reflection at $488 \mathrm{~nm}$ for five of the tapes used in this study are presented in Table I. For micron sized particles, one expects on the basis of Mie theory only a weak variation in the value of $g$ as the wavelength is changed. This was confirmed by analysis of the inline transmission 
using a $670 \mathrm{~nm}$ diode laser, where it was found that the calculated values of $\mathrm{g}$ at $670 \mathrm{~nm}$ ( 0.872 and 0.816 for the $\mathrm{CW}$ and $\mathrm{ZrO} 2$ loaded samples respectively) are similar to those at $488 \mathrm{~nm}$. We have now determined all the relevant optical parameters of the free standing scattering film, and thus we expect the radiative transport model to predict, without the use of any additional fitting parameters, the optical behavior for a wide variety of light input and output geometries. Thus, an independent test of the applicability of our model is a comparison of the calculated and measured angular dependence of the light scattered by the free-standing films. To perform such a test, a detector with radius $r=0.5 \mathrm{~cm}$ was mounted on a rotating arm of length $1=38.2 \mathrm{~cm}$ away from the scattering sample. A $670 \mathrm{~nm}$ laser beam was passed through the sample and intensity of the transmitted $670 \mathrm{~nm}$ light was then measured from $0^{\circ}$ to $85^{\circ}$. The measured intensity of the angularly deviated components is related to the calculated flux by a scaling factor $\mathrm{f}=5.4 \times 10^{-4}=\pi \mathrm{r}^{2} / 1^{2}$ which is the solid angle subtended by the detector. Using Eqn. 40:

$$
\begin{aligned}
& \frac{I_{670}(\varphi)}{I^{0}{ }_{670}}=f \Phi^{\text {out }}(\theta) \frac{d \Omega_{\text {int }}}{d \Omega_{e x}}=f \Phi^{\text {out }}(\theta) \frac{\cos (\varphi)}{n^{2} \cos (\theta)} ; \\
& \frac{I_{670}(0)}{I^{0}{ }_{670}}=\exp (-S)
\end{aligned}
$$

Since g, $\mathrm{n}$, and $\mathrm{S}$ are already determined, we can use the solution to Eqns 28-37 to determine $\Phi^{\text {out }}(\theta)$, and compare it to the measured values of the ratio on the left hand side without further adjustment. In the top panel of figure 10 we plot the angularly resolved intensity versus angle for one tape (CW particles at $5.6 \%$ weight loading). In addition, we plot the predictions of the model (Eqn. 43) for different input values of g. Clearly the curve generated using a value of $g=0.87$ provides an acceptable description of the data. It is possible to improve the fit to the data by introducing other phase function parameterization schemes ${ }^{43}$, and more accurately accounting for the averaging effects introduced by the finite size of the detection diode. To test this hypothesis, we also tried a two parameter model that seemed to provide a slightly better fit to the angular dependence data, however this model did not significantly affect our calculation of the total transmission and reflection characteristics of the film, and thus its use is not required.

\section{III.2.B.2. Optical Properties of OLEDs}

\section{III.2.B.2.A Effective Reflectance}

The reflectance of a typical OLED was measured using the specular reflectance attachment of a commercial spectrometer. A blue emitting OLED was manufactured using the procedure and materials described in section II.1. The reflectance spectrum was measured through the substrate and device (ITO+polymer) layers and is thus expected to be lower than the value of 0.91 obtained from calculating the reflectivity of a film of aluminum on glass. In Figure 11, we compare the experimental reflectivity spectrum to typical OLED spectra measured in an integrating sphere. Averaging over the output spectrum of the OLED device results in an effective reflectivity of 0.79 . In order to directly input this number into the radiative transfer model, the ideal OLED would be thin relative to the substrate area, and be uniformly reflective. The actual OLED tested had a 1" square active area OLED on a 1.5 " glass substrate of $1 \mathrm{~mm}$ thickness, however, 
most of the non-active area of the OLED was coated with an aluminum film that was separated from the ITO anode by a thin insulating layer, and only $7 \%$ of the device area was non-reflective. Since this area is relatively small, and non-emissive, it should have a minor effect on the total light output; thus we set $\mathrm{R}_{\mathrm{OLED}}=0.79$.

\section{III.2.B.2.B Angular dependence of OLED light emission}

The angular dependence of the OLED light output as it goes from the OLED layers into the substrate sets the initial condition $\mathrm{D}(\theta)$ in Eqn. 28. In principle, one could determine this internal angular distribution by measuring the external OLED light emission pattern using a methodology similar to our determination of the angular dependence of light scattering. This approach, however, requires deconvolution by the same ratio of solid angles that appears in Eqn. 40; this makes small changes in $D(\theta)$ hard to resolve. Thus, we set up a procedure to measure $\mathrm{D}(\theta)$ directly. In particular, we optically coupled the OLED to the center of a glass hemisphere and measured the light emitted as a function of angle from the hemisphere. ${ }^{10,14}$ This geometry effectively makes the ratio of solid angles equal to unity. In our experiments we place a small ( $1 / 4$ " square) OLED at the center point of a 3" diameter glass hemisphere. Two methods were used to probe the angular emission. In the first, an optical fiber input coupler was rotated around the hemisphere and the light coupled into the fiber was coupled to a remote detector. In order to minimize the effects of fiber misalignment an iris aperture and an optical diffuser were mounted before the fiber input. The second measurement method utilized a small silicon diode rather than the fiber input coupler as the rotating component. The integrated intensity as a function of angle utilizing both measurement methods is shown

Figure 12. The solid line is a calculated emission profile of the form $D(\theta)=\cos (\theta)^{1.2}$. The factor of 1.2 is determined by fitting the observed signal level in the diode versus $\cos (\theta)$ on a log-log plot.

\section{III.2.B.3 Light Output of OLEDs coupled to scattering films}

Given the complete characterization of the relevant properties of an OLED and a scattering film described above, the ultimate goal of this work is to predict the effect of coupling the scattering film to the OLED on light output. Accordingly, we define an enhancement factor, $\varepsilon$, as the total integrated light output from an OLED with the scattering tape, divided by the same quantity without the scattering tape. We measure this quantity in the following manner. The OLED was placed inside an 18 " diameter integrating sphere equipped with a fiber bundle leading to a $1 / 4 \mathrm{~m}$ spectrometer with CCD detection. The OLED was mounted using a 1/4" thick magnet on a white steel base. Thus, all light emerging in the forward hemisphere experience similar optical paths before detection. Light emerging from the sides of the OLED, traveling in the reverse direction, will strike the white base before being reflected into the sphere; some will be lost as a result of striking the back of the OLED, which is somewhat absorbing. A white light source $(10 \mathrm{~W}$, tungsten filament, previously calibrated against a NIST traceable blackbody source) was also mounted inside the sphere and provided an internal spectral calibration. The calibration was frequently checked and measured both with and without the OLED device in the sphere. Care was taken to ensure that the OLED was in a stable operating mode and that it was as clean as possible. A scattering tape was mounted on the on the surface of the OLED and the spectra measured. The tape was removed from the OLED and the measurement immediately repeated. We calculate the enhancement, $\varepsilon$, the 
ratio of I(scattering)/I(no-scattering) for each scattering film. In figure 13 we plot the enhancement factor (left axis) vs. the observed scatterance for tapes made out of both cool white phosphor particles (top) and $\mathrm{ZrO}_{2}$ particles (bottom). Error analysis indicates that the statistical uncertainty in the measurement of $\varepsilon$ is $+/-0.028$. The data is represented by the solid squares and error bars. Note that a maximum enhancement factor of 1.41 is observed. The predicted enhancement based upon the radioactive transport model and experimentally measured input parameters $(\mathrm{R}=0.79, \mathrm{z}=1.2$, $\mathrm{g}$ and $\mathrm{S}$ indicated in the figure) is shown as the solid line. The agreement between prediction and experimental data is quite impressive - particulary given the absence of any independently adjustable parameters in the model.

Given the enhancement factor, it is possible to calculate the absolute value of the extraction efficiency $\eta_{\mathrm{s}-\mathrm{a}}$ of an OLED coupled with scattering film if one knows the absolute value of the extraction efficiency $\eta_{\mathrm{s}-\mathrm{a}}$ of the bare OLED as follows:

$$
\eta_{s-a}=\eta_{s-a}^{0} \varepsilon
$$

In order to get an accurate estimate of $\eta_{\mathrm{s}-\mathrm{a}}$ one must consider finite size effects on an actual device. The primary effect of size is that some of the emission from the OLED occurs from the sides of the device. This has the effect of increasing the total outcoupling efficiency relative to an infinite device where no emission can come from the sides. Thus, the out-coupling efficiency that can be calculated analytically for an infinitely large OLED provides a lower limit for $\eta_{\mathrm{s}-\mathrm{a}}$.

$\eta_{s-a}^{0}>\int_{0}^{\theta_{c}} 2 \cos ^{z}(\theta) \sin \theta d \theta$

Assuming a substrate with index $n=1.5, \theta_{c}=41.8$, and our measured value of $z=1.2$, eq. 23 predicts a lower limit for $\eta_{\mathrm{s}-\mathrm{a}}$ of 0.469 . A better estimate for $\eta_{\mathrm{s}-\mathrm{a}}$ can be obtained using ray-tracing calculations with the actual OLED geometry as well as its mount inside the integration sphere. Such calculations were performed and gave an estimate for $\eta_{\mathrm{s}-\mathrm{a}}$ of $0.534+/-0.035$ (ASAP version 7.1). This number, combined with eq. 22 was used to calculate the absolute extraction efficiency from the measured enhancement values. These are depicted as the right-hand axes in figure 5 . Note that a maximum output coupling efficiency of 0.75 is observed with the cool-white phosphor.

\section{III.2.B.4. Discussion}

The data and model presented in figure 13 can be described using a simple physical picture. In both data sets, there is an optimal value for the film scatterance, $\mathrm{S}$. At low values of scatterance, waveguiding within the scattering medium is not completely suppressed, while at very high values of scatterance, the light is reflected from the scattering medium back to the lossy cathode. The peak value is the point where these two effects are balanced. According to our modeling, the position of this optimum value depends upon the value of $\mathrm{g}$. The data in figure 13 clearly show this trend, as the optimal amount of scatterance in $\mathrm{ZrO}_{2}$ loaded films is $\sim 2$, and the optimal amount for CW loaded films is $\sim 3$. More importantly, we observe a high level of agreement between the analytical model and the data, both in terms of the magnitude of the light output 
enhancement, and the trends in the light output as the scatterance and the phase function of the scattering particles are varied. Considering both the experimental error in our determination of the model input parameters, the possible errors in the determination of the absolute outcoupling efficiency are $\sim \pm 7 \%$.

To summarize our methodology: we have presented an experimental determination of the parameters that are needed to calculate the extraction efficiency $\eta_{\mathrm{s}-\mathrm{a}}$ and measurements of $\eta_{\mathrm{s}-\mathrm{a}}$ in OLED devices that are optically coupled to different scattering films. We first determine the scattering parameters, $S$ and $g$, for each film that will be coupled to the OLED device, the OLED reflectivity (R RLED $_{\text {) and internal angular }}$ light distribution $(\mathrm{D}(\theta))$. Finally, we couple each film to the OLED and measure the enhancement factor, $\varepsilon$, which we define as the ratio of the number of photons with the scattering tape divided by the number of photons measured from the bare OLED. There is a linear relationship between $\varepsilon$ and $\eta_{\mathrm{s}-\mathrm{a}}$ that connects our observations to the model and permits a detailed assessment of the model's accuracy and capabilities. Our analysis requires no free adjustable parameters, and the high level of agreement between fundamental physical models and detailed physical measurements provides a large degree of confidence in our estimate of the absolute extraction efficiency

In conclusion, our results demonstrate that it is possible to obtain a quantitative measurement and prediction of the increase in light output due to volumetric light scattering, and we demonstrate a clear increase in the observed extraction efficiency. We find that for an OLED on a glass substrate the fraction of light that crosses the substrateair interface increases to almost 0.75 . Using ray-tracing, we are able to account for the differences between the analytical infinite plane model and the observed results, we require no additional parameters to model our data. The high level of agreement between the model and the data serves to validate the use of simple radiative transport models to predict OLED efficiencies and optimization strategies. While in the experiments described in this paper examine only the contributions volumetric light scattering to total light output, in the companion paper we also discuss the effects of microcavities and describe simple extensions of the model to describe the effect of surface texturing. Thus, having validated our radiative transport model, we may extend it to an uniform description and quantitative assessment of many different enhancement and color tuning schemes that have appeared in the literature. 


\section{Achieving Large Area - A New Architecture ${ }^{44}$}

A key obstacle to the development of large area OLEDs is the presence of local defects which cause electrical shorts. Some shorts are relatively benign in that they "burn-out" during operation resulting in only a small non-emissive, non-conducting area where the short was. ${ }^{45}$ However, some do not burn out and some develop over time. ${ }^{46,47}$ These are catastrophic since current flows through the short rather than the working areas of the device. This is true even for pixelated device architectures when all pixels are simultaneously energized as would be required for lighting applications. Causes of shorting defects include particle contamination during fabrication, asperities from electrode roughness, and nonuniformities in organic layer thickness. ${ }^{45,46,47}$ For all of these mechanisms, the chance of encountering a defect increases as device area increases. This is problematic for lighting applications where device sizes on the order of square meters are envisioned.

Another obstacle to achieving large area devices results from the fact that OLEDs are current-driven, ie. brightness scales with current density. Thus, larger devices require a greater current to be spread throughout the active area. There is a resistance to this spreading due to the finite conductivity of the electrodes which can be quantified in terms of the voltage drop $\left(\mathrm{V}_{\mathrm{d}}\right)$ as current travels along the relevant length $(\mathrm{L})$ of the active area. Assuming a rectangular emitting geometry, it is straightforward to show that this voltage drop can be expressed approximately in terms of the average brightness (B), current efficiency $(\varepsilon)$ and electrode sheet resistance $\left(R_{s}\right)$ as follows:

$$
V_{d} \cong R_{s} L^{2} \frac{B}{\varepsilon}
$$

In order to maintain efficiency and brightness uniformity, $V_{d}$ should ideally be significantly less than the intrinsic voltage $V_{i}$ required across the thickness of a device to attain a specific brightness. The electrode sheet resistance of a typical OLED is dominated by that of the indium-tin-oxide (ITO) transparent conductor to a value of $\sim 10$ ohms/square. The brightness required for most lighting applications is on the order of $1000 \mathrm{~cd} / \mathrm{m} 2$. The most efficient OLEDs demonstrated to date at this brightness are green devices and these require an intrinsic voltage of $\sim 4 \mathrm{~V}$ and have a current efficiency of $\sim 70$ $\mathrm{cd} / \mathrm{A}^{48}$ Substituting these values into eq. 46 , one can see that, even for the most efficient OLEDs, the current spreading length has to be less than $\sim 5 \mathrm{~cm}$ to keep $V_{d}$ less than $10 \%$ of $\mathrm{V}_{\mathrm{i}}$. Again, this is a significant issue when device sizes on the order of square meters are envisioned.

In this letter, we demonstrate an OLED device architecture that addresses both of these obstacles to large area devices. Our approach, illustrated schematically in figure 14, consists of dividing the desired large area device into separate smaller emitting elements connected monolithically in series. With this approach, the required current is controlled by the individual element area rather than by the total emitting area of the device. Hence, $L$ can always be chosen to be small enough to minimize $V_{d}$ relative to $\mathrm{V}_{\mathrm{i}}$. Similarly, the series connection addresses the problem of catastrophic shorts. Such 
shorts still occur but, with this design, their deleterious effect should be isolated to the particular element that they are associated with while the required current continues to flow through the rest of the device. Hence the device as a whole still emits light efficiently - albeit with a non-emitting area corresponding to the size of the shortcircuited element. It should be noted that this approach to fault-tolerance is passive and does not require expensive sensing or switching circuitry.

In order to demonstrate this architecture, three 5-element and six 12-element series-connected light-emitting polymer (LEP) based devices were fabricated on a single substrate according to the layout depicted in figure 14. The fabrication was as follows. ITO coated glass was patterned using standard photolithographic techniques and cleaned with an oxygen plasma. A thin $(60 \mathrm{~nm})$ film of PEDOT/PSS (Poly $(3,4)$ ethylendioxythiophene / polystyrene sulfonate) - polymer obtained from Bayer Corporation was immediately deposited atop the ITO via spin casting and then baked for one hour at $200{ }^{\circ} \mathrm{C}$. A polyfluorene-based blue LEP obtained from Cambridge Display Technologies was then spin coated atop the PEDOT/PSS layer to a thickness of about 60$70 \mathrm{~nm}$. At this point, a $\mathrm{KrF}$ excimer laser operating at $248 \mathrm{~nm}$ was used to selectively ablate away the polymer layers and expose ITO between neighboring elements in order to enable the required anode to cathode electrical connections. A cathode consisting of $4 \mathrm{~nm}$ of $\mathrm{NaF}$ followed by $200 \mathrm{~nm}$ of $\mathrm{Al}$ was then thermally evaporated through a shadow-mask such that, for each element, the cathode extended beyond the active area to make contact with the exposed ITO from the neighboring element (figure 14). Finally, the substrate was removed into an inert atmosphere and the OLED layers were sealed to a top cover glass for encapsulation.

The performance of each device was tested by placing the substrate in an integrating sphere and measuring the total optical power output as a function of voltage with a calibrated photodiode. The optical spectrum was also measured which enabled a direct calculation of the average brightness assuming lambertian emission. Figure 15 summarizes the device performance of a representative 5- and 12-element device from the substrate along with the average performance of a "single element" OLED with the same layer structure and an active area of $0.26 \mathrm{sq} . \mathrm{cm}$. One can see from part A of the figure that the voltage required to attain a particular brightness scales approximately with the number of elements in series as expected for this architecture. Figure 15B shows the electrical to optical power efficiency of these devices plotted versus the current density through each element. The error bar represents the spread in efficiency values for the "single-element" device type over multiple fabrication runs. It is clear that this random error is much larger than any differences between the efficiency curves. Hence, the efficiency obtained with this series architecture does not appear to depend on the number of elements in series and is indistinguishable from the "intrinsic" efficiency of the smallarea single-element device.

In order to test fault tolerance, 20 substrates consisting of twelve 12-element with individual element areas of $1.2 \mathrm{~cm}^{2}$ were made on $15.2 \times 15.2 \mathrm{~cm}$ substrates. Extensive care was not taken to prevent manufacturing defects as various process steps where the organic layers were exposed were not performed in a cleanroom environment. Most 
substrates had at least one device without any manufacturing defects and these had performance comparable to that of the smaller element-area 12-element device depicted in figure 15. However, it was often found that at least one device on a substrate contained a non-emissive element. All such devices still emitted light from the remaining elements indicating that the defect causing the dark element was a catastrophic short. Presumably, if all the elements were pixels operating in parallel, the whole device would have been non-emissive. The fact that 11 of the 12 elements still emit demonstrates the fault-tolerance of this monolithic series architecture. It should be noted that completely nonemissive devices were never observed indicating that shorts, rather than open-circuits are likely the dominant defect type.

It is instructive to compare the performance of devices with a short to those without a short on the same substrate. Typically, the intact elements of a device containing a short exhibit higher brightness than the elements of a defect-free device at the same applied voltage. This indicates a higher average voltage across each intact element which implies that the resistance of the short is considerably less than the dynamic resistance of each element. Such a condition is required if the series architecture is to maintain efficiency. Figure 16 depicts the efficiency of the devices with one shorted element divided by the same quantity averaged over the completely intact devices on the same substrate. One can see that, even in the presence of these normally catastrophic shorts, for $90 \%$ of the devices, efficiency is maintained at a level within approximately $20 \%$ of that of the comparable defect-free devices.

An ideal fault-tolerant architecture should result in minimal perturbation in device performance in the presence of defects. In principle, this ideal can be approached by dividing the desired emitting area into more, and smaller, series connected elements. In this way, a catastrophic short can result in an unnoticeably small non-emissive area while the average increase in voltage across each intact element - and hence brightness change is minimized. This strategy is limited by the increasing fabrication complexity required as element and interconnect sizes are decreased. For example, when low-cost, web-based fabrication techniques are desired, the minimum element size will likely be determined by the patterning capability of the technique such as ink-jet, gravure, or screen used to print the active organic layers. Ultimately, this tradeoff between degree of fault-tolerance and fabrication complexity will always exist and needs to be evaluated when designing a device.

Theoretically, it is possible to scale up the fault-tolerant architecture described here to make a single, monolithic, 2'x2' OLED. Ultimately, this may be the best approach in commercial production but, due to equipment limitations, it was decided to build the final deliverable 2'x2' OLED lighting panel, a tiling approach would be adopted. Hence, the final deliverable consists of sixteen 6" 6 " OLED devices with the design shown in figure 14 and utilizing the white light downconversion approach discussed in section II. 
Table 1: Experimental and scatterance, total transmission and total reflectance data for 5 different particle loadings in PDMS. Data was collected at $488 \mathrm{~nm}$. The value of $\mathrm{g}$ needed to best match the data using the radiative transfer model is shown in the last column, along with the calculated values of total transmittance and reflectance.

\begin{tabular}{|l|l|c|c|c|c|c|}
\hline Scatterer & S & T (expt) & R (expt) & T (model) & R (model) & g (model) \\
\hline ZrO$_{2}$ & 1.91 & 0.770 & 0.231 & 0.77 & 0.23 & 0.79 \\
\hline ZrO $_{2}$ & 3.38 & 0.684 & 0.314 & 0.684 & 0.315 & 0.798 \\
\hline ZrO $_{2}$ & 5.62 & 0.581 & 0.426 & 0.578 & 0.42 & 0.777 \\
\hline 'cool white' & 3.77 & 0.736 & 0.254 & 0.742 & 0.256 & 0.877 \\
\hline 'cool white' & 6.90 & 0.631 & 0.364 & 0.633 & 0.366 & 0.872 \\
\hline
\end{tabular}


Figure 1: Blue device emission spectrum (A) and emission (dotted lines) and absorption spectra (solid lines) of the downconversion materials - Lumogen ${ }^{\mathrm{TM}}$ orange (B) and red (C), and Y(Gd)AG:Ce (D) - used to construct the white device. Panel E shows the emission spectra of the white device (dotted line) along with the fit (solid line) to a downconversion model. Absorbance and Emission Intensity are in arbitrary units.

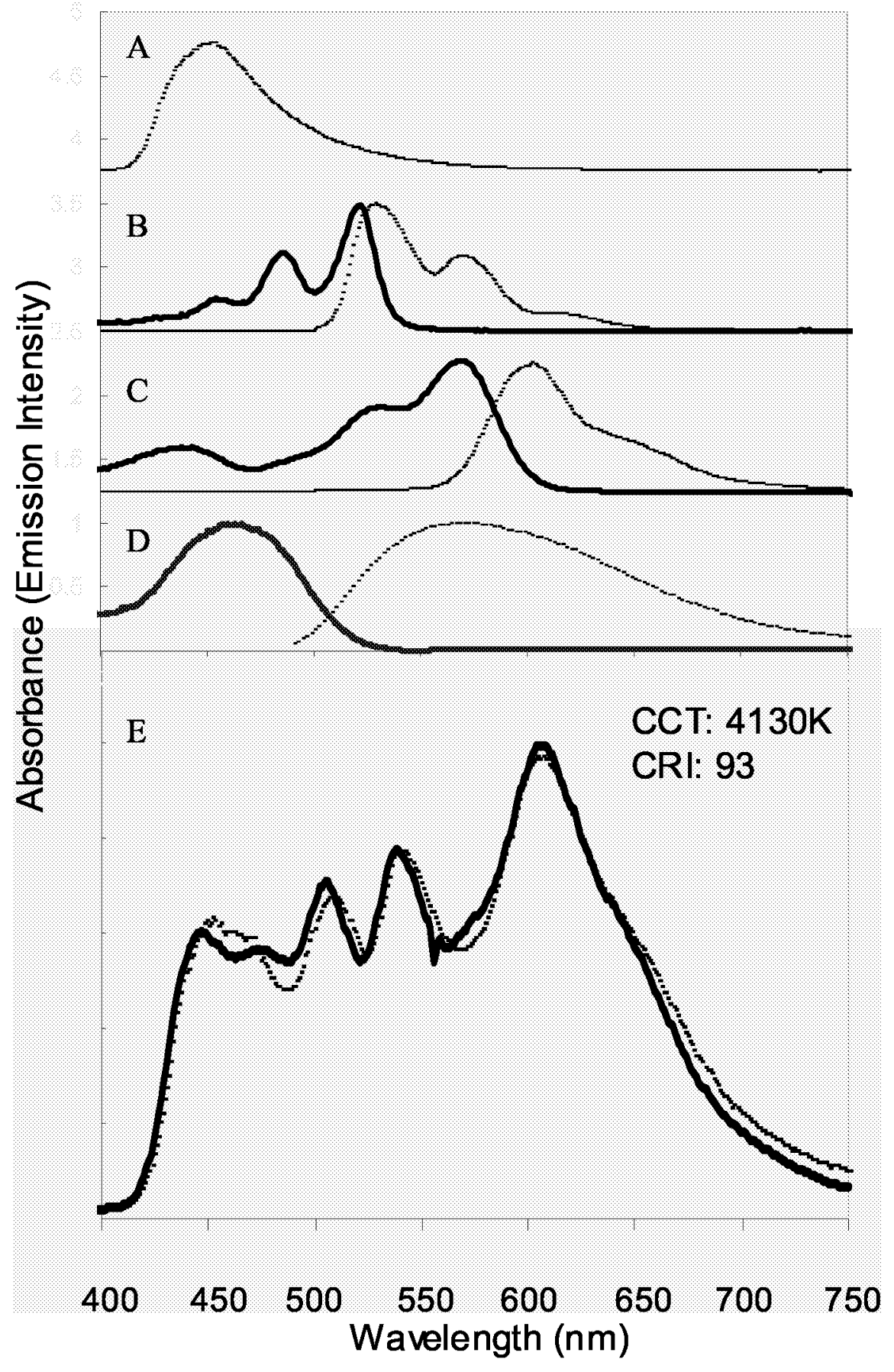


Figure 2: Top: Brightness vs. applied voltage for a blue (filled circles) and white (open circles) device. The inset tabulates device performance at $5.5 \mathrm{~V}$, where the white device emits $1080 \mathrm{~cd} / \mathrm{m}^{2}$. The white model is the calculated performance based upon the blue device efficiency and the expected downconversion losses. Bottom: Percent power conversion efficiency vs. voltage. The solid line represents the expected white efficiency based upon the model.
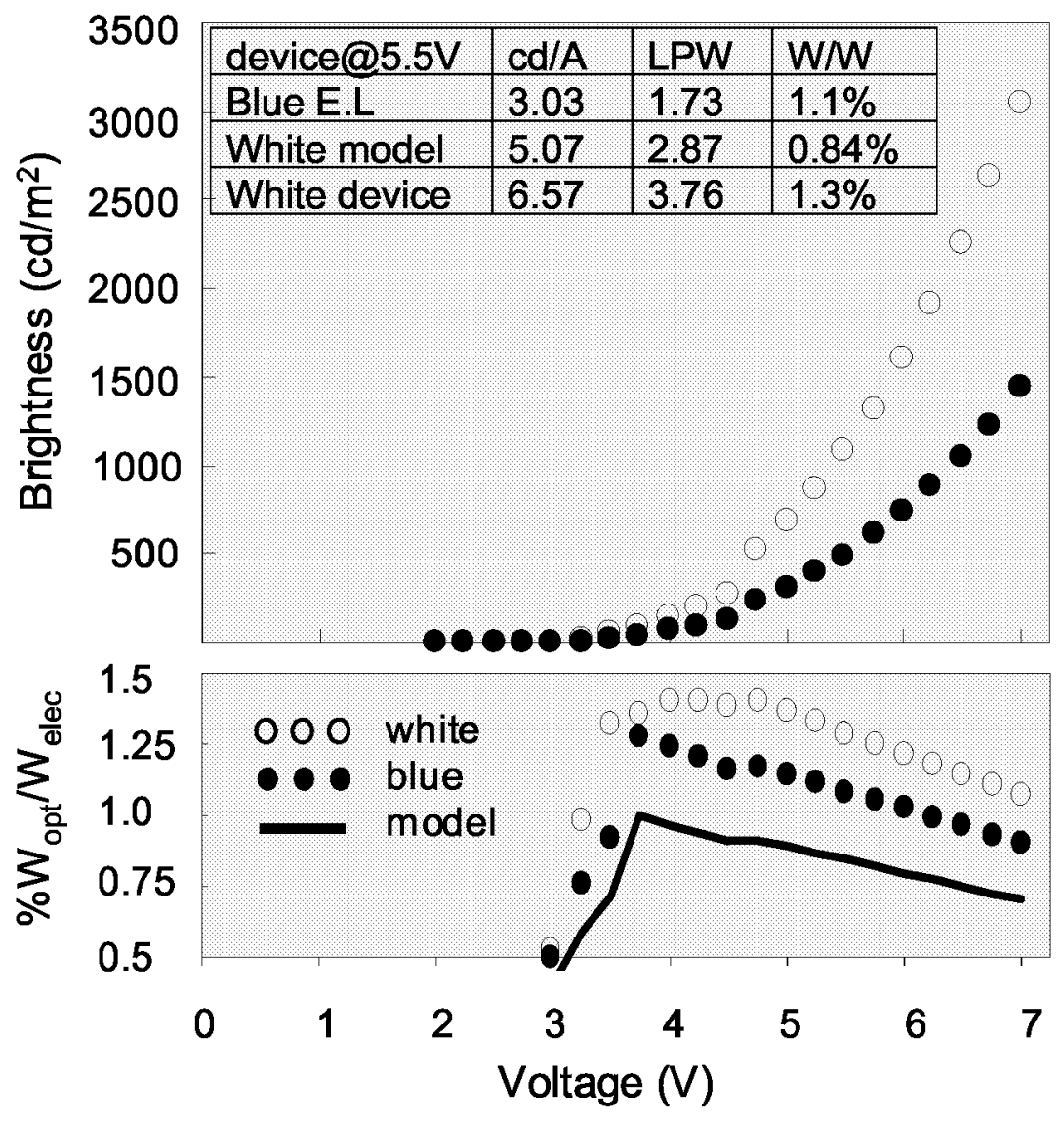
Figure 3: Schematic illustration of an OLED device (top) and the idealized model geometry and model input parameters (bottom).

\section{Device}

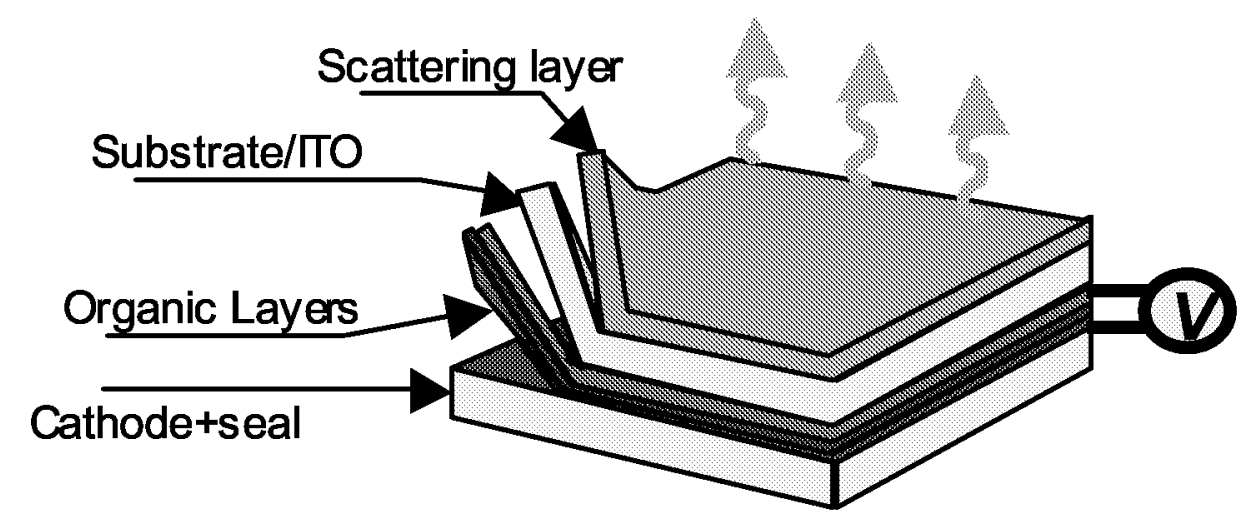

Model

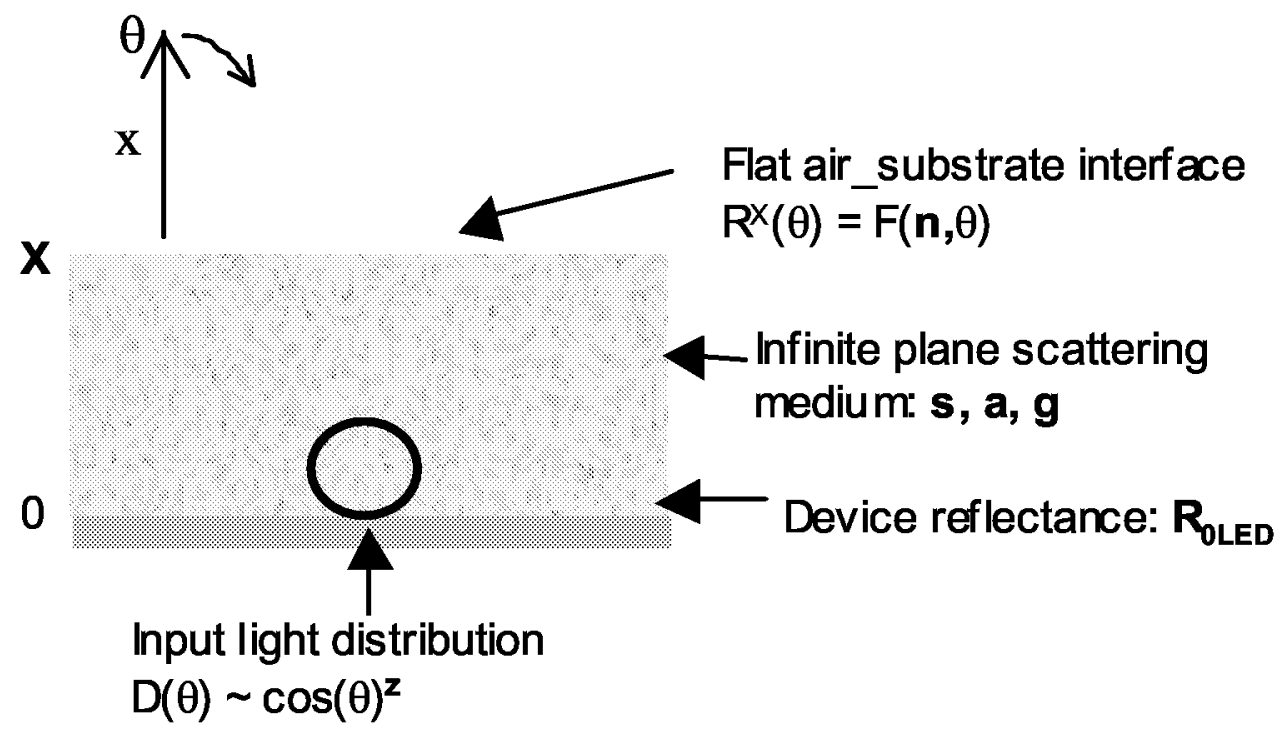


Figure 4: Calculated substrate to air extraction efficiency, $\eta_{\mathrm{s}-\mathrm{a}}$ as a function of scatterance assuming different values of the cathode reflectivity. The input light distribution was assumed to have a Lambertian profile, the index, $\mathrm{n}$, was set equal to 1.5 and the asymmetry parameter, $\mathrm{g}$, was set equal to 0.8

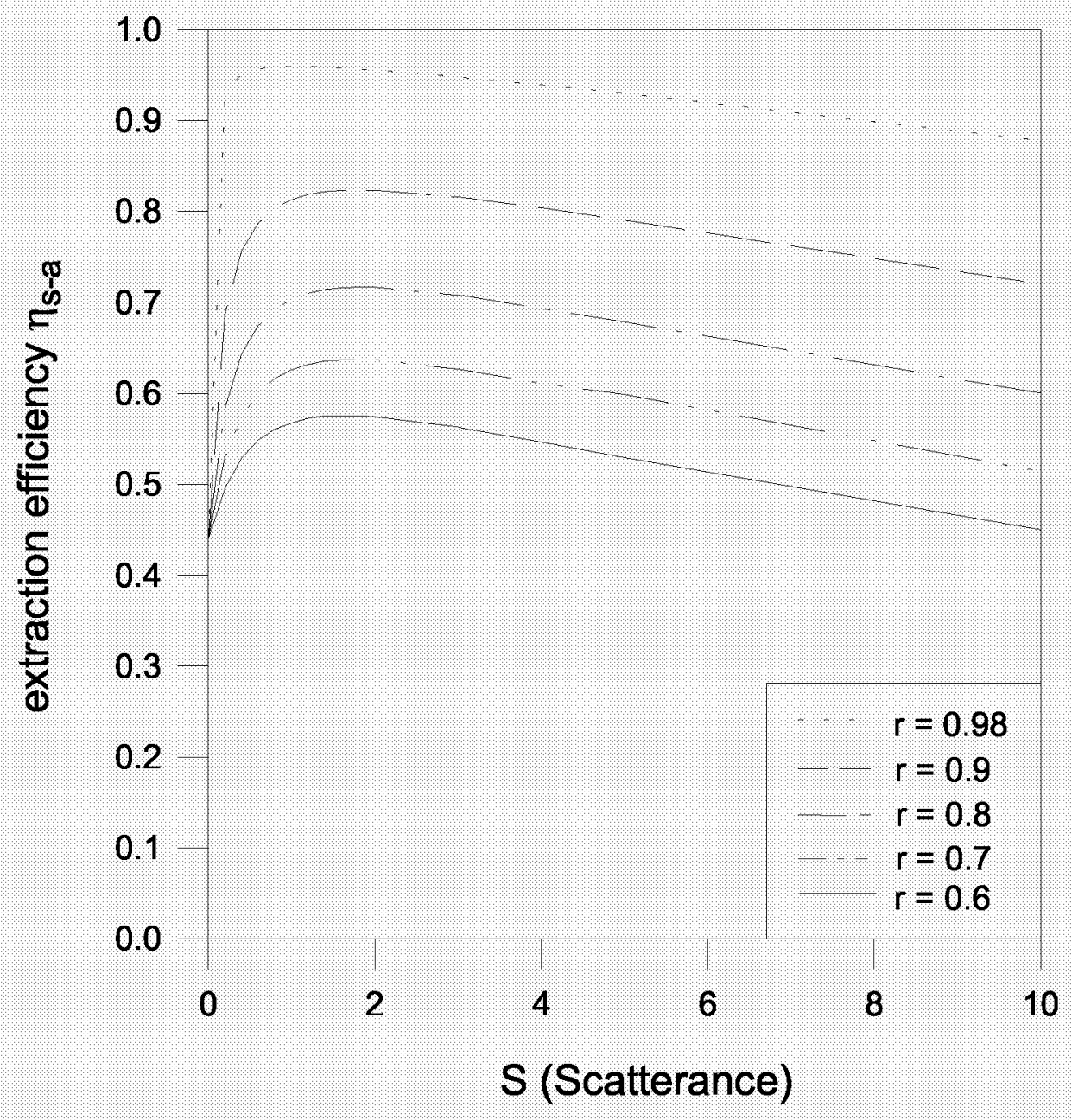


Figure 5: Calculated enhancement of OLED light output as a function of scatterance for different values of the asymmetry parameter $\mathrm{g}$. The optical index $\mathrm{n}$, was set equal to 1.5 and the cathode reflectance was set to 0.8 .

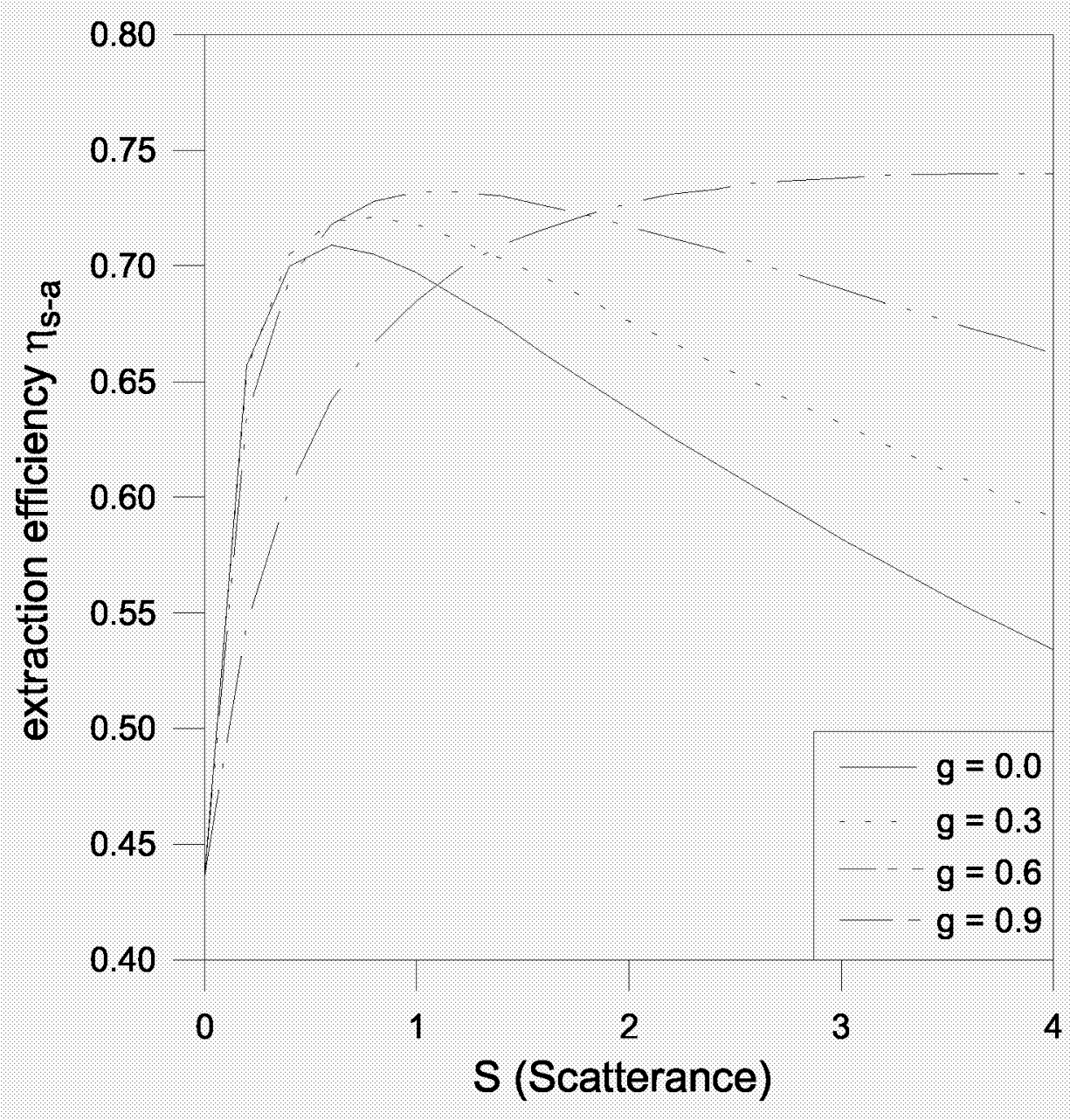


Figure 6: Calculated substrate to air extraction efficiency, $\eta_{\mathrm{s}-\mathrm{a}}$ assuming optimum values of the scatterance as a function of substrate index of refraction. The different curves assume different values for the substrate index.

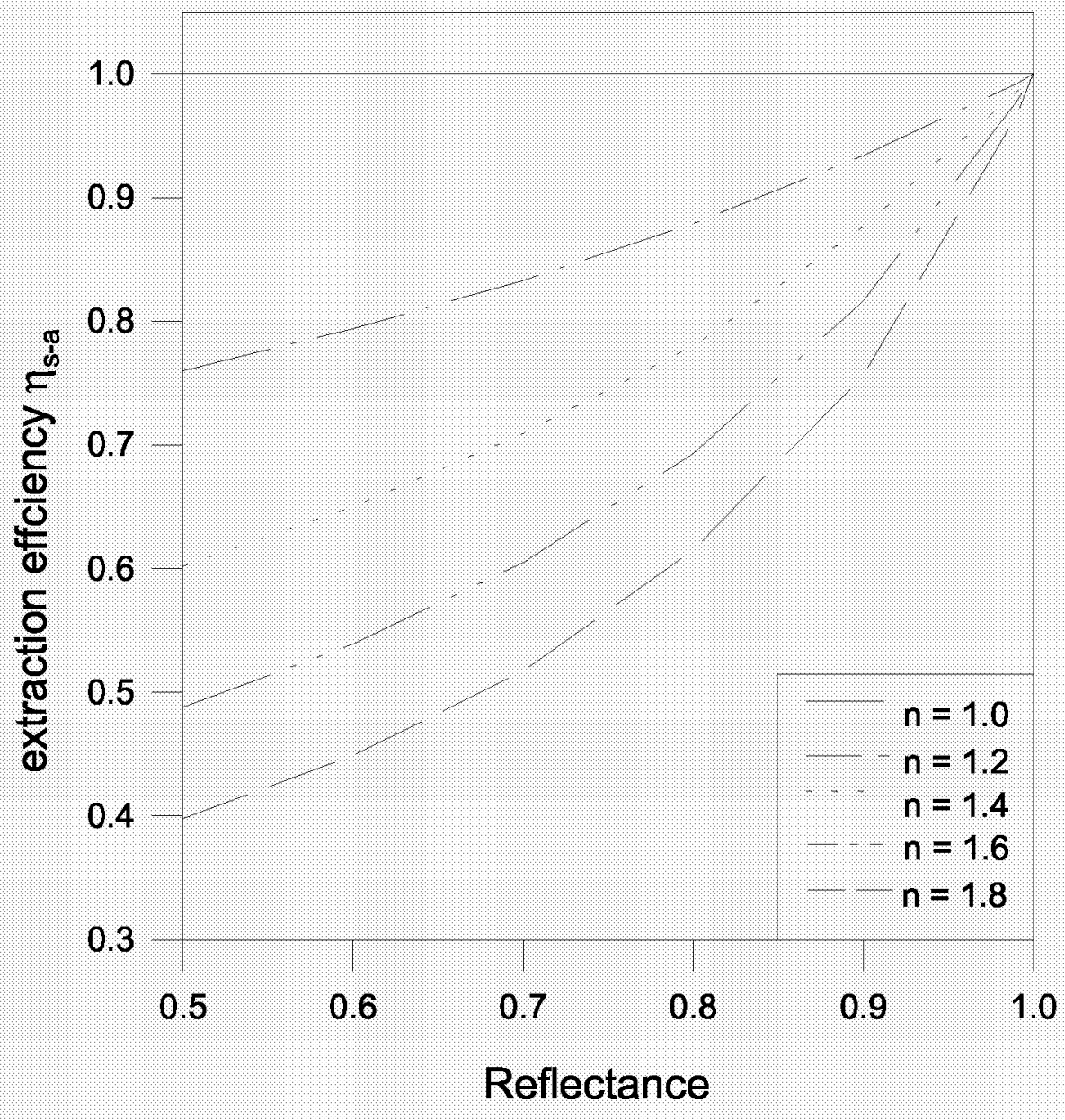


Figure 7: Calculated substrate to air extraction efficiency, $\eta_{\mathrm{s}-\mathrm{a}}$ assuming optimum values of the scatterance as a function of substrate index of refraction for two different angular emission profiles. The assumed cathode reflectivity was 0.79 , the anisotropy, $g$, was 0.9.Inset: plot of angular emission profiles for different values of $\mathrm{z}$ (solid lines). For comparision, the polarization averaged results of a calculation using a microcavity model of a standard OLED structure are shown assuming both isotropically oriented dipoles (squares) and dipoles oriented parallel to the plane of the OLED (circles).

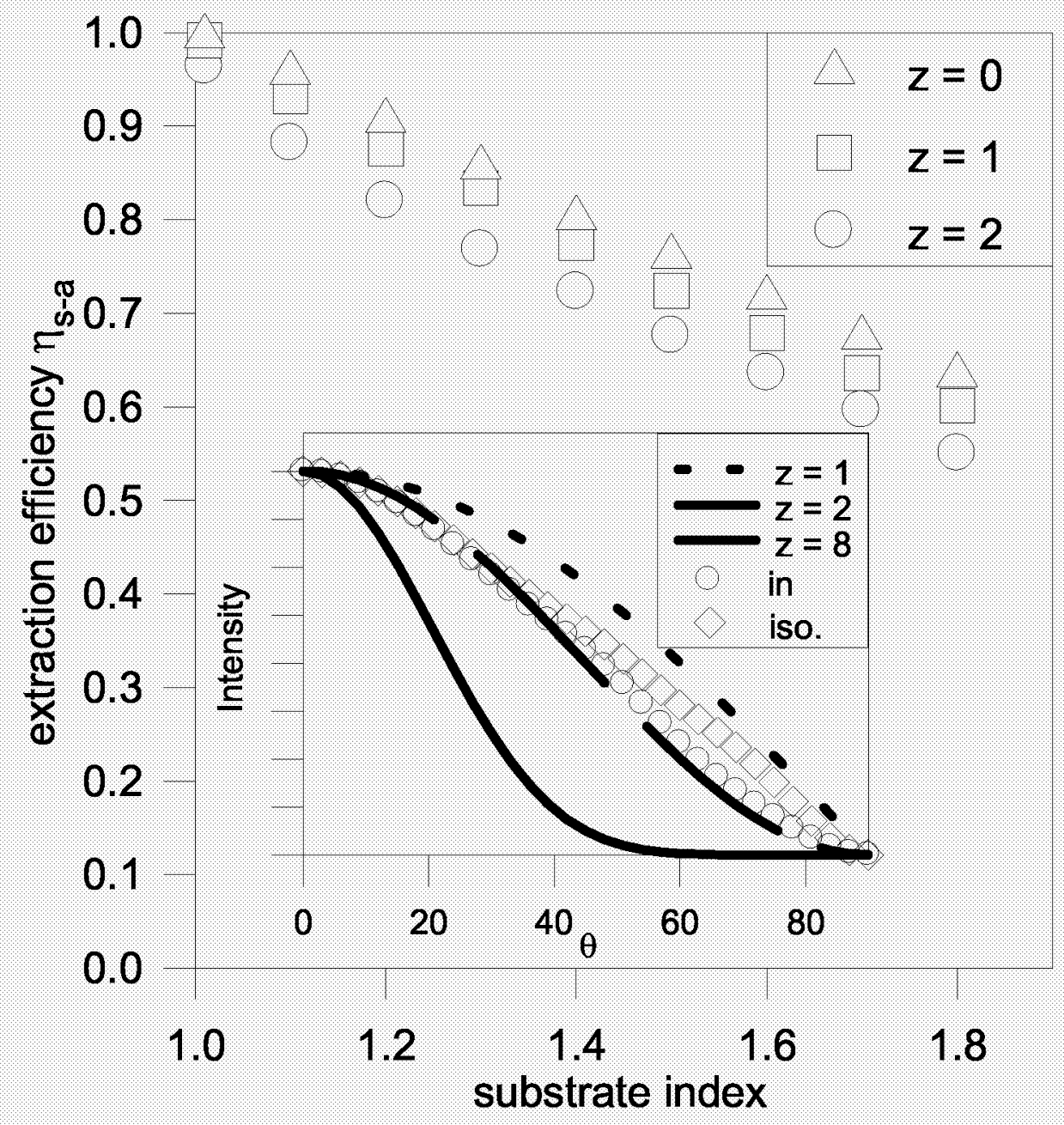


Figure 8: Contour plot showing the variation of extraction efficiency $\eta_{\mathrm{s}-\mathrm{a}}$ as a function of both the scatterance and the angular emission profile. The optical index of the substrate was 1.5 and the anisotropy, g, was equal to 0.8 .

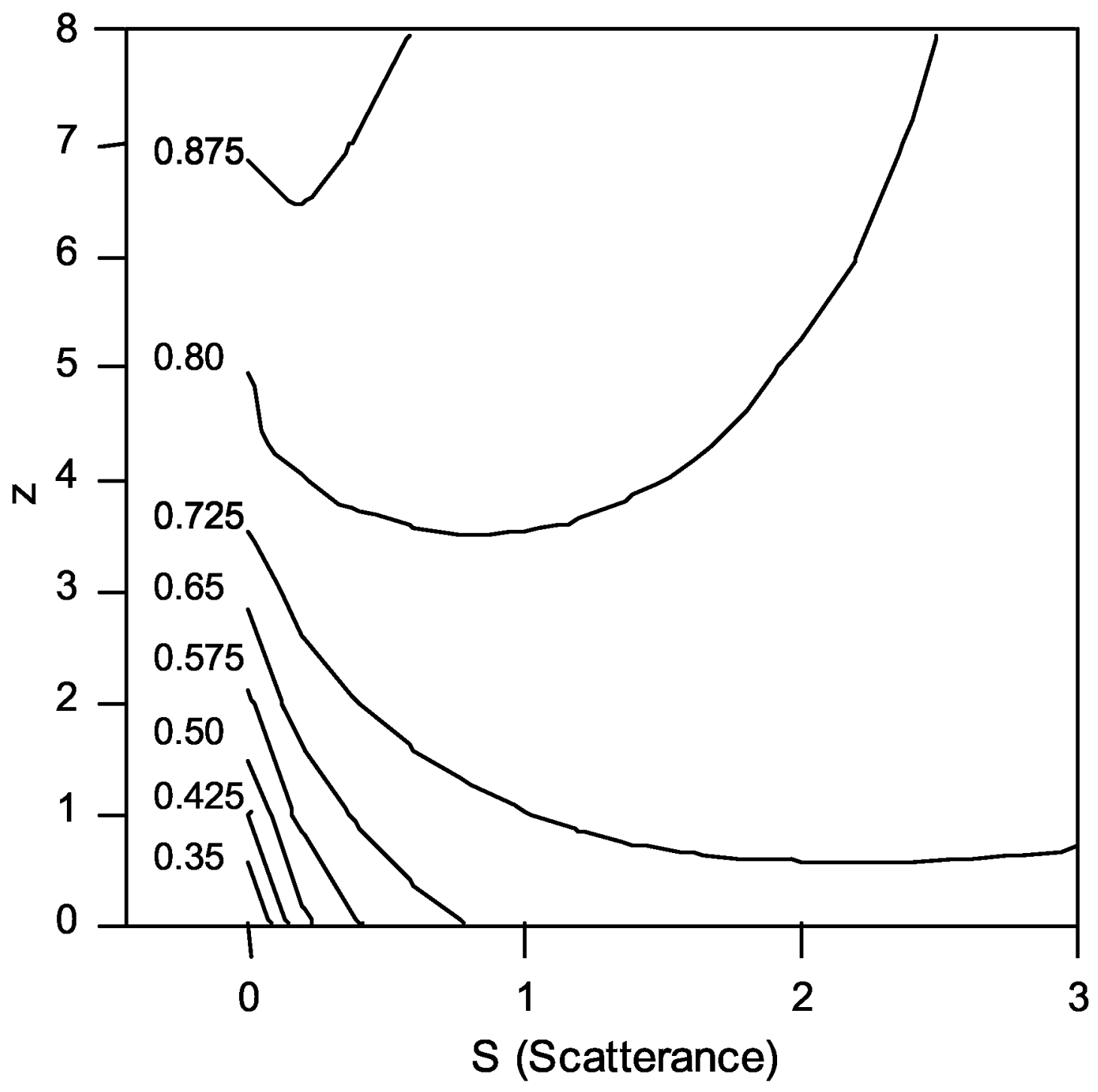


Figure 9. A schematic illustration of the different physical processes which affect transport of light in a scattering medium. The line represents the history of a single prototypical ray whose interactions with the medium and scattering sites (labeled points $\mathrm{a}-\mathrm{g}$ ) are described in the text.

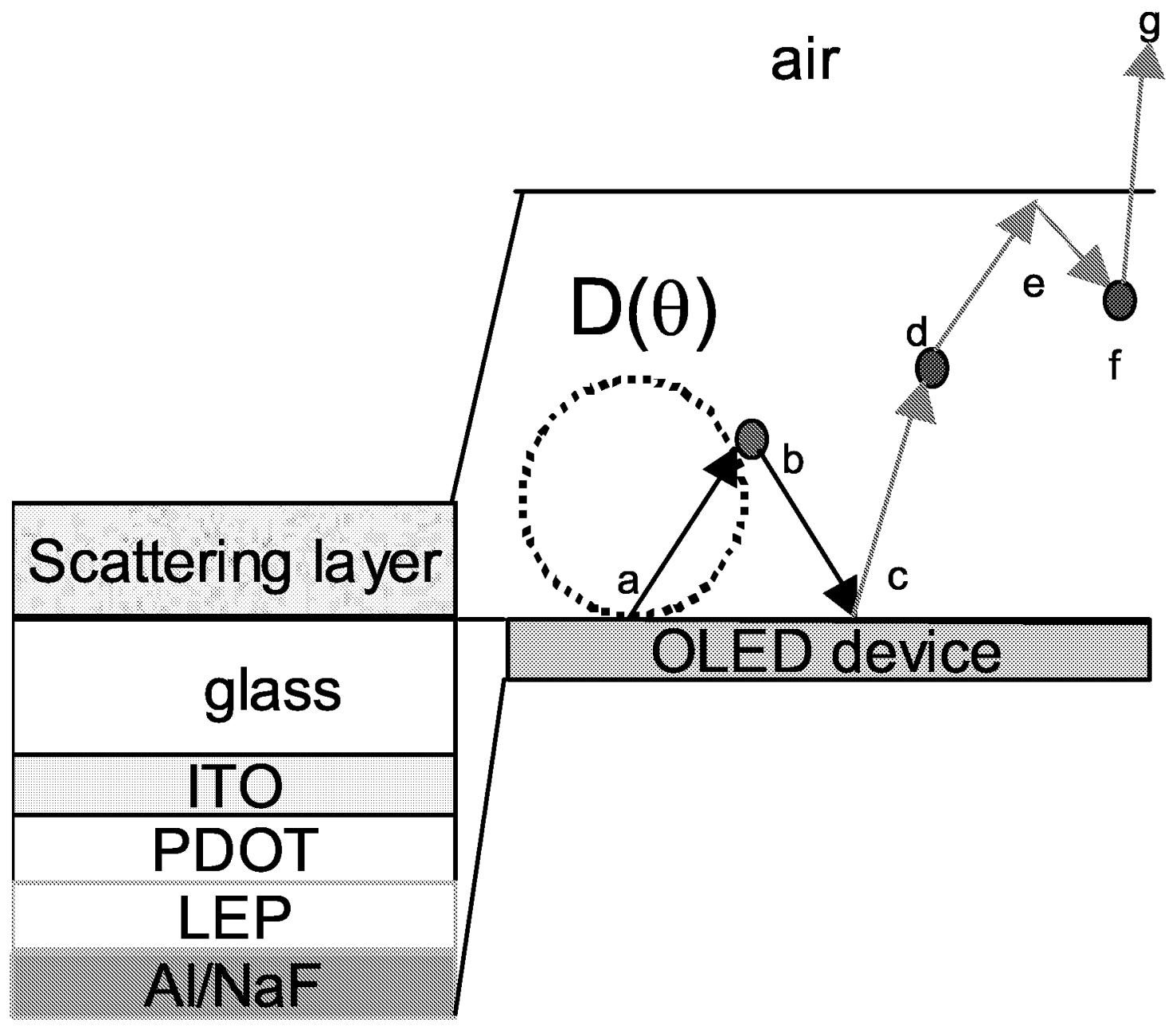


Figure 10. Measured angular dependence of light scattering of a collimated $670 \mathrm{~nm}$ beam incident on a PDMS tape loaded with 'cool white' phosphor particles (open circles). The solid line is the radiative model prediction using the H-G phase function (Eqn.4) using $\mathrm{g}=0.87$, derived from fitting the data in Table I.

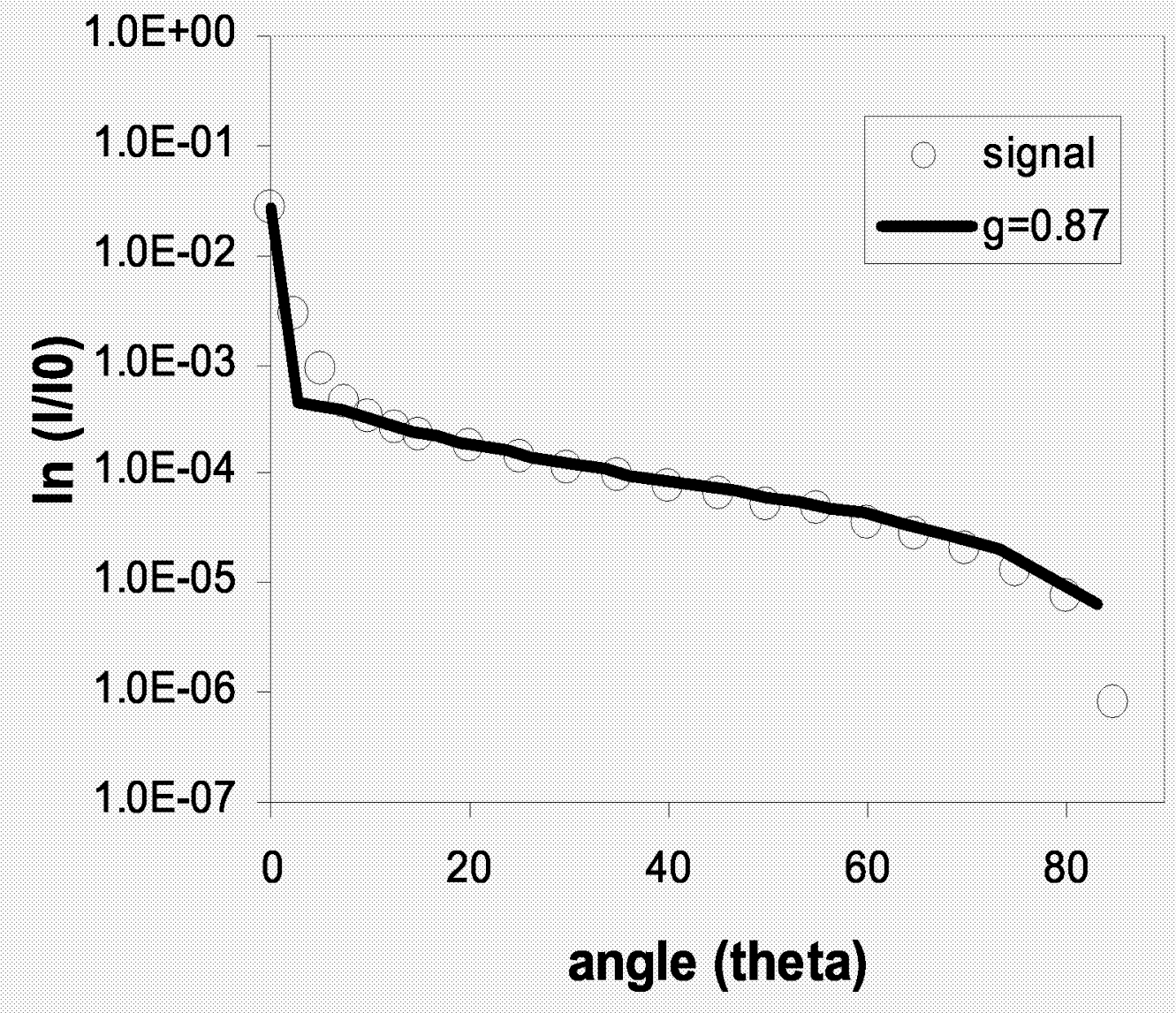


Figure 11. Measured specular reflectivity of OLED device (right y axis) and typical blue OLED spectra measured in an integrating sphere (left y axis).

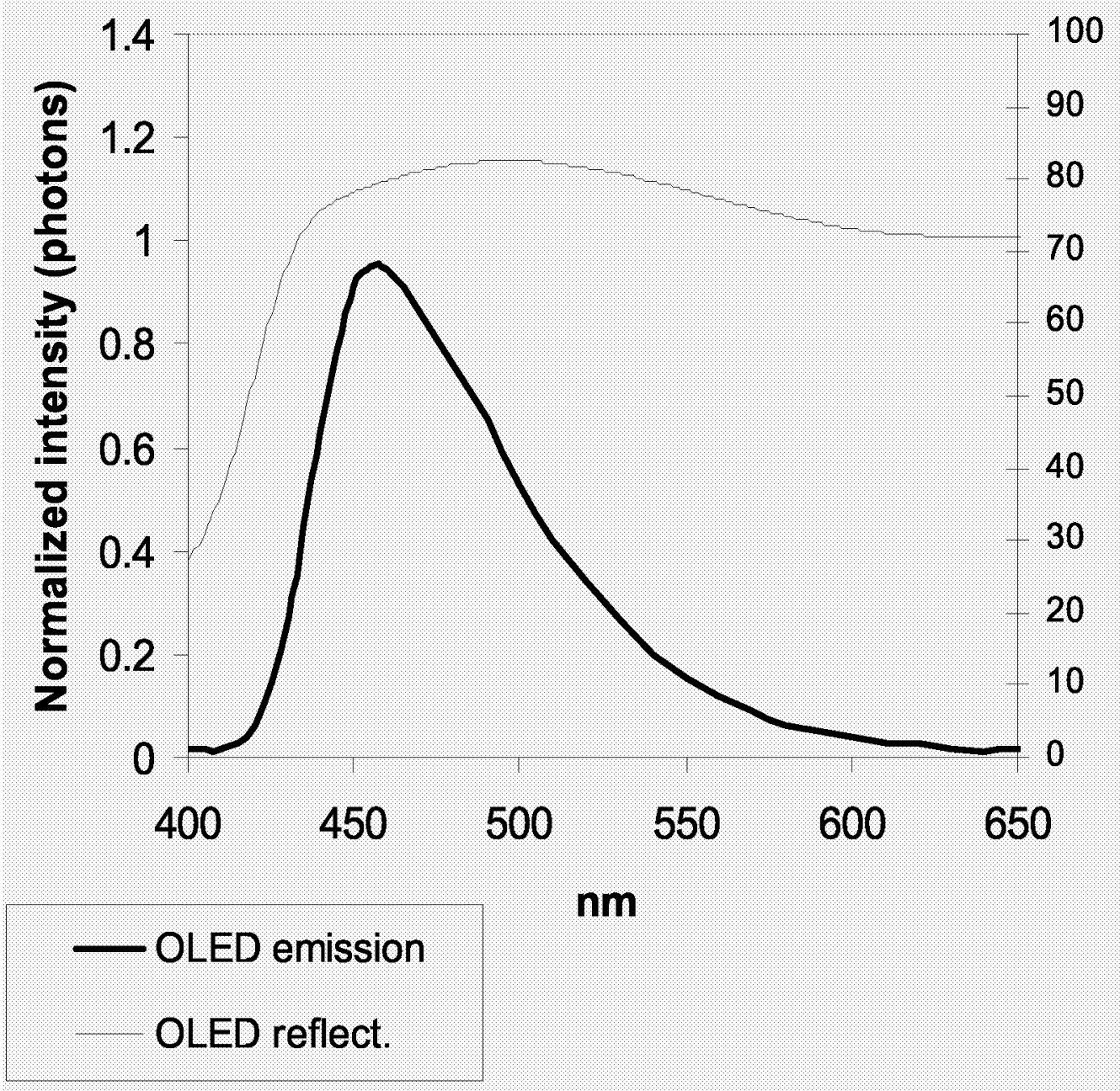


Figure 12. Angular distribution of light intensity emitted by the OLED into the glass substrate, as measured using a glass hemisphere optically coupled to the OLED. $0^{\circ}$ is the direction normal to the OLED surface. The data are acquired using both a Si detector (solid diamonds) and a fiber coupled CCD camera (open squares) and are compared to the function $\cos (\theta)^{1.2}$ (solid-line)

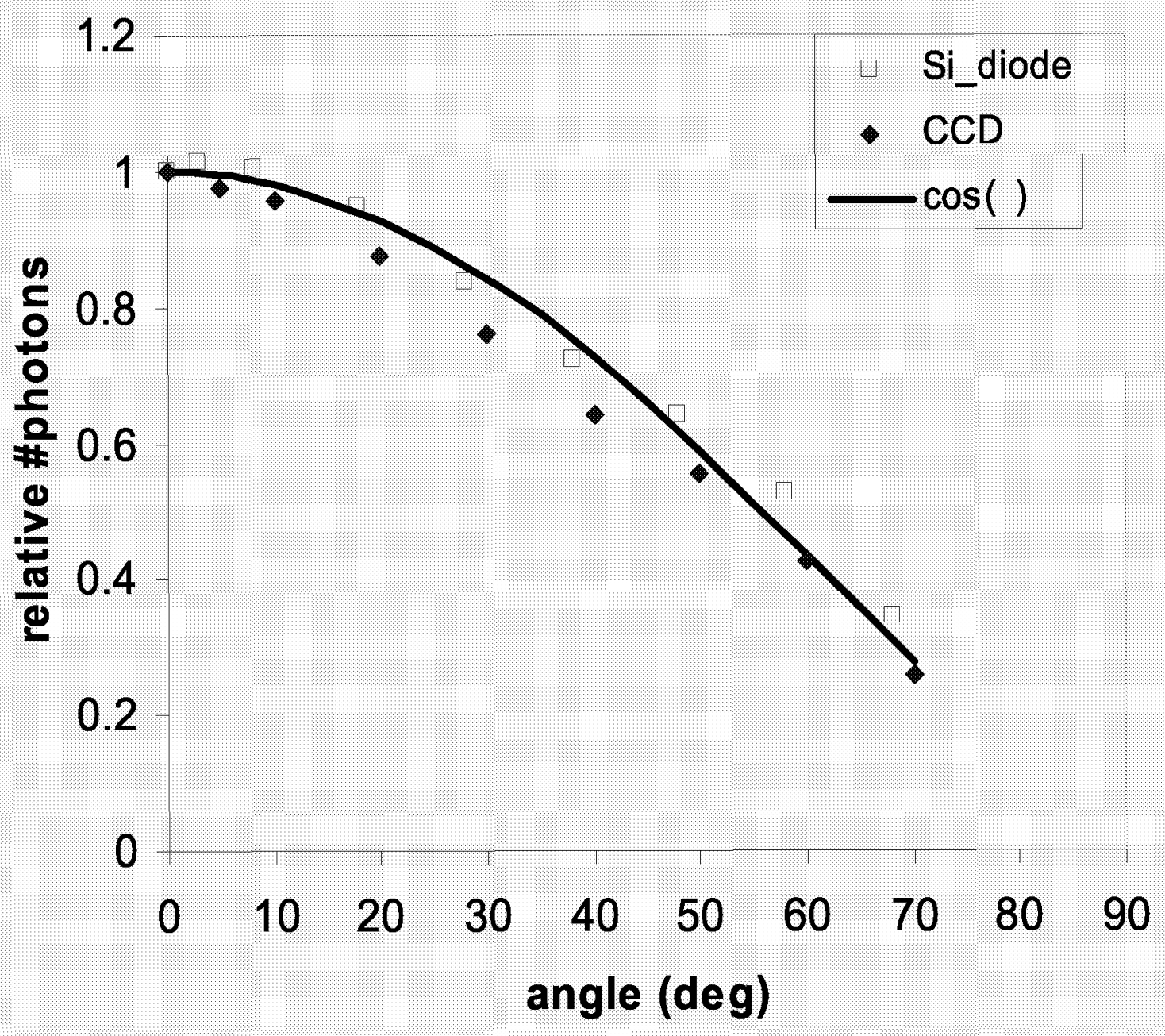


Figure 13. Light output enhancement as a function of scatterance in the substrate. The left $y$-axis is the observed ratio of the OLED output when coupled to the scattering tape divided by the bare OLED output. The right y-axis is the calculated air-substrate extraction efficiency, $\eta_{\mathrm{s}-\mathrm{a}}$. Top: data obtained using PDMS tapes loaded with 'cool white' phosphor particles. The solid line is the output of the model with $g=0.87$. Bottom: data obtained using PDMS tapes loaded with $\mathrm{ZrO}_{2}$ particles. The solid line is the output of the with $\mathrm{g}=$ 0.79
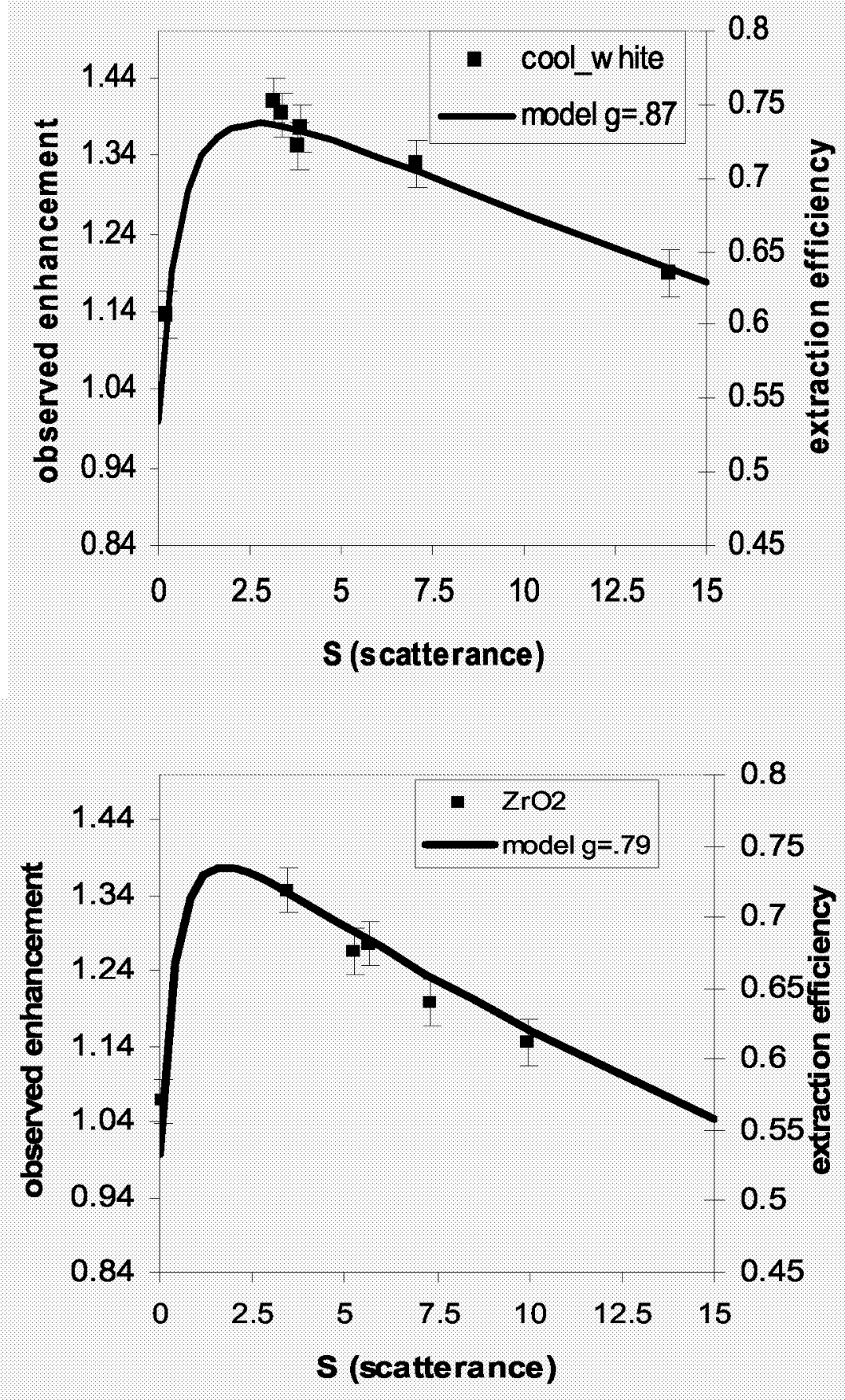
Figure 14: (Top) Schematic side-view representation of the element interconnect structure of the proposed monolithic series architecture with 3 elements in series. The active area of each element is defined by the region where the organic layers are sandwiched between ITO and cathode metal. (Bottom) Scale top view of anode (horizontal hash) and cathode (diagonal hash) layout of substrate containing six 12element and three 5-element devices. The active areas of each individual element of the 12 and 5 element devices are $0.28 \mathrm{~cm}^{2}$ and $1.2 \mathrm{~cm}^{2}$ respectively.
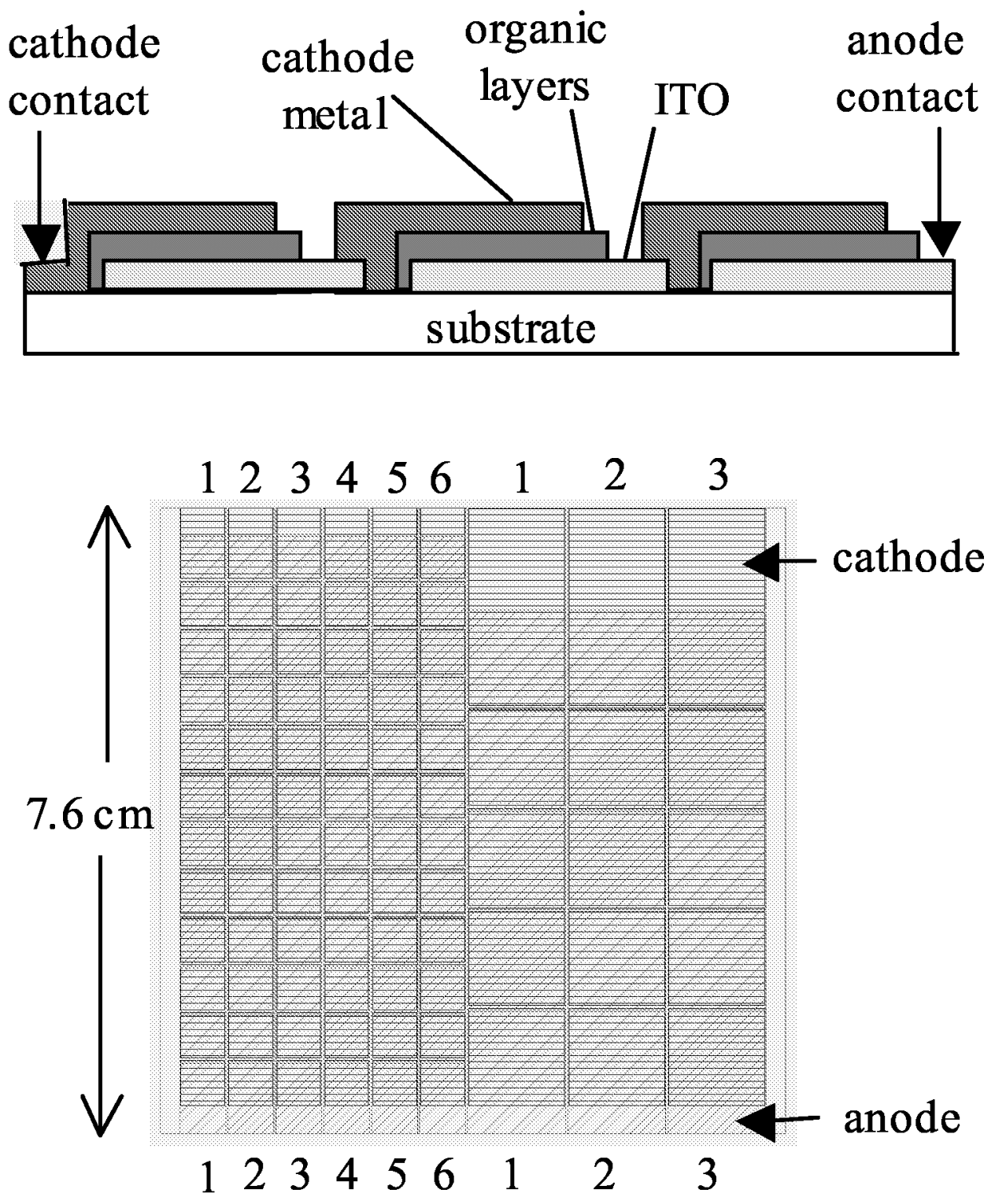
Figure 15: Brightness vs. voltage (A) and power efficiency vs. current density (B) data of devices made with 5 and 12 elements in series on the same substrate compared with average data from a comparable single element device.

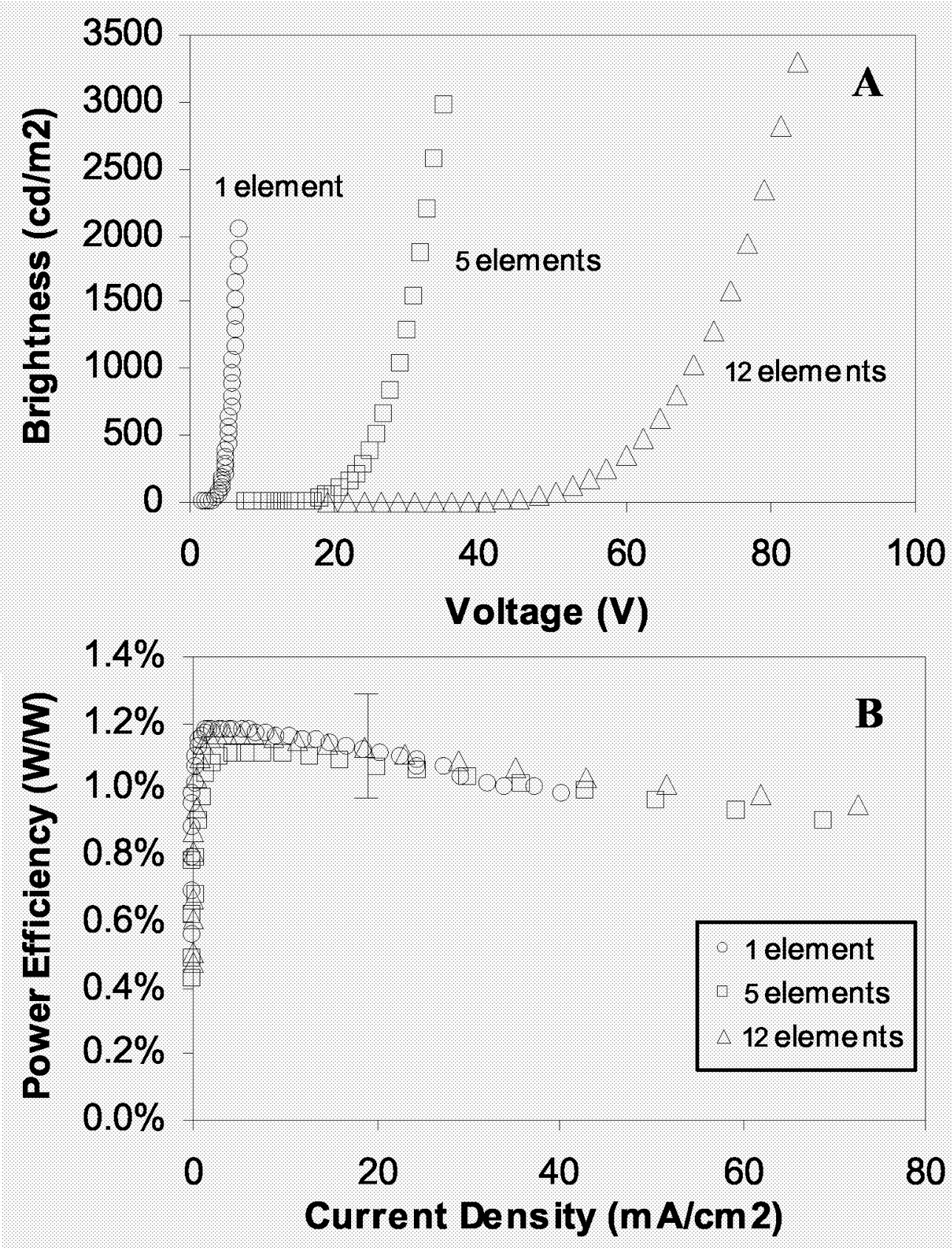


Figure 16: Power efficiency of devices containing a catastrophic short normalized to that of defect-free devices from the same substrate. Note that 26 of the 29 defective devices still maintain power efficiency to within $20 \%$ of the defect-free value.

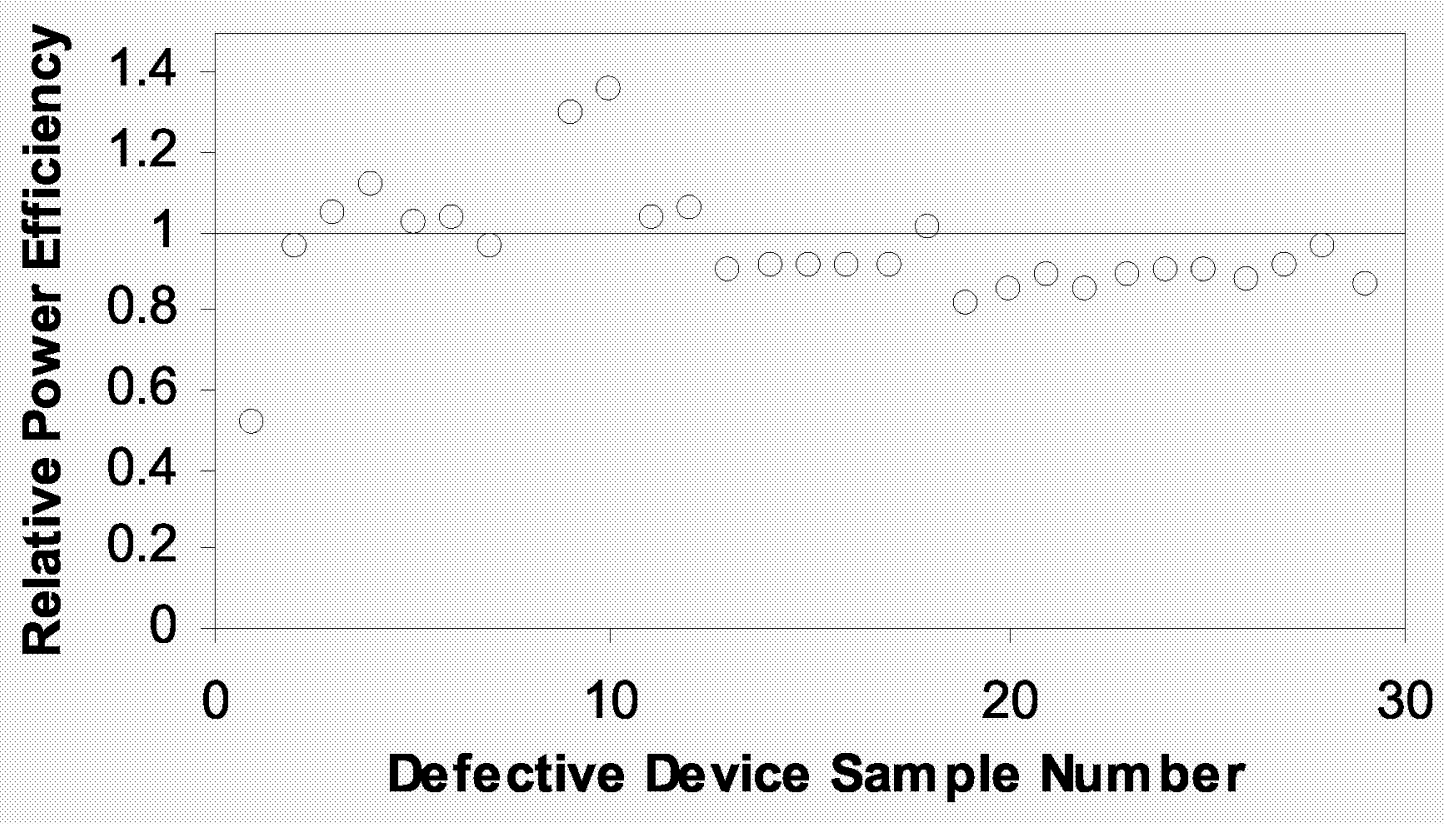




\section{References}

${ }^{1}$ A. R. Duggal, J. J. Shiang, C. M. Heller, and D. F. Foust, Appl. Phys. Lett., 80, 3470 (2002).

${ }^{2}$ G. Wyszelki, W.S. Stiles, Color Science ${ }^{\text {nd }}$ Ed.,(John Wiley and Sons, New York,1982)

${ }^{3}$ J. Kido, M. Kimura, K. Nagai, Science 2671332 (1995).

${ }^{4}$ J. Kido, H. Shionoya, and K. Nagai, Appl. Phys. Lett. 672281 (1995).

5 J. Thompson, R. I. R Blyth, M. Mazzeo, M. Anni, G. Gigli, and R. Cingolani, Appl. Phys. Lett. 79560 (2001).

${ }^{6}$ W.H. Melhuish, J. Phys. Chem 65, 229, (1961)

${ }^{7}$ H. I. Schnitzer, E. Yablonovitch, C. Caneau, T. J. Gmitter, and A. Scherer, Appl. Phys. Lett. 632174 (1993).

${ }^{8}$ J.J. Shiang and A. R. Duggal, J. Appl. Phys. ,95 2880 (2004)

9 J. J. Shiang, T. J. Faircloth, and A. R. Duggal, J. Appl. Phys. ,95,2889 (2004)

${ }^{10}$ J.-S. Kim,P. K. H. Ho, N. C. Greenham, R. H. Friend, J. Appl. Phys. ,88 ,1073 (2000)

${ }^{11}$ M. H. Lu, J.C. Sturm, J. Appl. Phys., 91, 595, (2002)

${ }^{12}$ K.Meerholz, D.C. Müller, Adv. Functional Mater.,11,251, (2001)

${ }^{13}$ W.N. Carr, Infrared Physics, 6, 1, (1966)

${ }^{14}$ V. Bulovic, V.B Khalifin, G. Gu, P.E. Burrows, D.Z. Garbuzov, and S.R. Forrest, Phys. Rev. B., 58, 3730, (1998).

${ }^{15}$ G. Gu, D. Z. Garbuzov, P.E. Burrows, S. Venkatesh, S. R. Forrest, M. E. Thompson, Optics Letters, 22, 396, (1997)

${ }^{16}$ T. Tsutsui, N. Takada, S. Saito, E. Ogino, Appl. Phys. Lett., 65, 1868, (1994)

${ }^{17}$ H. Benisty, H. De Neve, C. Weisbuch, IEEE J. of Quantum Electron., 34, 1612, (1998)

${ }^{18}$ S. Tokito, T. Tsutsui, Y. Taga, J Appl. Phys, 86, 2407 (1999).

${ }^{19}$ T. Tsutsui, M. Yahiro, H. Yokogawa, K. Kawano, M. Yokoyama, Adv. Mat., 13, 1149, (2001) 
${ }^{20}$ R. Windish, P. Heremans, A. Knobloch, P. Kiesel, G.H. Dohler, B. Dutta, G. Borghs, Appl. Phys. Lett, 74, 2256, (1996)

${ }^{21}$ H.I. Schnitzer, E. Yablonovitch, C. Caneau, T. J. Gmitter, and A. Scherer, Appl. Phys. Lett. 63, 2174, (1993)

${ }^{22}$ C.F. Madigan, M. H. Liu, J.C. Sturm, Appl. Phys. Lett, 76, 1650, (2000)

${ }^{23}$ S. Möller, S. F. Forrest, J. Appl. Phys., 91 ,3324, (2002)

${ }^{24}$ J. M. Lupton, B.J. Matterson, I.D.W. Samuel, M.J. Dory, W.L. Barnes, Appl. Phys. Lett, 77, 3340, (2000)

${ }^{25}$ M. Boroditsky, T.F. Krauss, R. Coccioli, V. Vrijen, R. Bhat, E. Yablonovivh, Appl. Phys. Lett., 75, 1036, (1999)

${ }^{26}$ T. Yamasaki, K. Sumioka, T. Tsutsui, Appl. Phys. Lett, 76, 1243, (2000).

${ }^{27}$ H.C. Van de Hulst, Multiple Light Scattering, (Academic Press, New York, 1980)

${ }^{28}$ pg. 128, H.C. Van de Hulst, Light Scattering by Small Particles, (Dover, New York, 1981)

${ }^{29}$ Born, Wolf, Principles of Optics, (Pergammon Press, Oxford, 1980)

${ }^{30}$ T. F. Soules, W. A. Klatt, J. Illum. Eng. Soc., 17, 92, (1988)

${ }^{31}$ Y.X. Hu, B. Wielicki, B. Lin, G. Gibson, S.C.Tsay, K, Stamnes, T. Wong, J. Quant., Spect. Rad. Trans, 65, 681, (2000)

${ }^{32}$ M. I. Mishchenko.;L. D. Travis,;;J. W. Hovenier, Light scattering by Nonspherical Particles : theory, measurements and applications. (Academic Press, San Diego, 2000)

${ }^{33}$ L.G. Henyey, J. L. Greenstein, Astrophys. J., 93,70, (1941)

${ }^{34}$ W.M. Cornette, J.G. Shanks, Appl. Optics, 31, 3152, (1992)

${ }^{35}$ S. Chandrasekhar, Radiative Transfer, (Dover, New York, 1960)

${ }^{36}$ P.S. Mudgett, L.W. Richards, Appl. Optics, 10, 1485, (1971)

${ }^{37}$ K. Stamnes, S.C. Tsay, W. Wiscombe, K. Jayaweera, Appl. Optics, 27, 2502, (1988)

${ }^{38}$ J. Gruner, F. Cacialli, R. H. Friend, J. Appl. Phys. 80, 207, (1996)

${ }^{39}$ O. H. Crawford, J. Chem. Phys., 89, 6017, (1988) 
${ }^{40}$ L.G. Henyey, J. L. Greenstein, Astrophys. J., 93,70, (1941)

${ }^{41}$ H.C. Van de Hulst, Multiple Light Scattering, (Academic Press, New York, 1980)

${ }^{42} \mathrm{~A}$ web based calculation tool and additional references are also available at http:/omlc.ogi.edu/calc/index.html

${ }^{43}$ W. M. Cornette, J.G. Shanks, Appl. Optics, 31, 3152, (1992)

${ }^{44}$ A. R. Duggal, D. F. Foust, W. F. Nealon, C. M. Heller, Appl. Phys. Lett., 82, 2580 (2003).

${ }^{45}$ P. E. Burrows, V. Bulovic, S. R. Forrest, L. S. Sapochak, D. M. McCarty, and M. E. Thompson, Appl., Phys., Lett., 65, 2922 (1994).

${ }^{46}$ H. Antoniadis, M. R. Hueschen, J. McElvain, J. N. Miller, R. L. Moon, D. B. Roitman, J. R. Sheats, Polym. Preprint., 38, 382 (1997).

${ }^{47}$ X. Zhou, J. He, L. S. Liao, M. Lu, X. M. Ding, X. Y. Hou, X. M. Zhang, X. Q. He, and S. T. Lee, $A d v$. Mater. 12, 265 (2000).

${ }^{48}$ M. Ikai, S. Tokito, Y. Sakamoto, T. Suzuki, and Y. Taga, Appl. Phys. Lett., 79, 156 (2001). 